\title{
Warm dust as a tracer of galaxies with gaseous halos
}

\author{
M. Dahlem ${ }^{1}$, J. S. Lazendic ${ }^{2,3}$, R. F. Haynes ${ }^{3}$, M. Ehle ${ }^{4,5}$, and U. Lisenfeld ${ }^{6}$ \\ 1 Sterrewacht Leiden, Postbus 9513, 2300 RA Leiden, The Netherlands ${ }^{\star}$ \\ 2 Astrophysics Department, School of Physics A28, University of Sydney, NSW 2006, Australia \\ 3 Australia Telescope National Facility, PO Box 76, Epping, NSW 2121, Australia \\ 4 XMM-Newton Science Operations Centre, Apartado 50727, 28080 Madrid, Spain \\ 5 Astrophysics Division, Space Science Department of ESA, ESTEC, 2200 AG Noordwijk, The Netherlands \\ ${ }^{6}$ IRAM, Avenida Divina Pastora 7, NC, 18012 Granada, Spain
}

Received 20 December 2000 / Accepted 1 May 2001

\begin{abstract}
We present radio continuum observations conducted with the VLA and ATCA of a sample of 15 edgeon spiral galaxies. 11 of these galaxies, with inclination angles of $i \gtrsim 75^{\circ}$ and neither active galactic nuclei nor nearby interaction partners, are suitable for studies of halo properties in relation to the level of star formation in their disks. In 6 of these 11 galaxies radio halos were detected at the angular resolution of the current data. In the remaining cases the presence of halo emission could not be proven unambiguously, partly due to relatively low angular resolution. A clear trend was found that galaxies with radio halos are those with the highest far-infrared $60 \mu \mathrm{m}$ to $100 \mu \mathrm{m}$ flux ratios. This shows the suitability of high $f_{60} / f_{100}$ ratios of $\geq 0.4$ as a reliable tracer of galaxies with high star formation rates and related disk-halo interactions, leading to the presence of extraplanar emission, e.g. from cosmic ray electrons. The measured exponential scale heights of those 6 radio halos that were clearly detected range from about 1.4 to $3.1 \mathrm{kpc}$. All 4 physically small galaxies in our sample do show extraplanar synchrotron radio emission, indicating that their more shallow gravitational potential compared to normal-sized spirals might facilitate the escape of cosmic-ray electrons from the sites of star formation in their disks. Although the galaxies with the highest energy input rates into the ISM of their disks are those that have the most prominent radio halos, there is no direct relation between the halo scale heights and the energy input rates. Instead, the scale heights of the radio halos are dominated by the energy losses of the cosmic ray electrons on their way out of the galaxy disks.
\end{abstract}

Key words. ISM: general - galaxies: evolution - galaxies: halos - galaxies: starburst - radio continuum: galaxies

\section{Introduction}

Despite accumulating evidence for the existence of gaseous halos around a number of nearby spiral galaxies (see, e.g., Dettmar 1992; Dahlem 1997 for overviews), it is currently a matter of controversy how many and exactly which galaxies have such halos. While the existence of gaseous halos (including radio halos, i.e. cosmic ray electrons) seems to be in general a rather rare phenomenon (Hummel et al. 1991a found that only about $5.5 \%$ of the 181 galaxies in their initial optically selected sample show signs of extraplanar radio emission), their existence appears to be quite common among nearby galaxies with high star

\footnotetext{
Send offprint requests to: M. Dahlem,

e-mail: mdahlem@eso.org

* Present address: ESO, Alonso de Cordova 3107, Vitacura, Casilla 19001, Santiago 19, Chile.
}

formation rates (SFRs; e.g. Dahlem 1997). We find that this apparent discrepancy is caused by two factors.

First, different selection criteria were used to define the sample of candidate galaxies. Hummel et al.'s objects were selected by their optical (blue) magnitude. However, especially when studying edge-on systems (in searches for halos), the blue magnitude is not always a good indicator of the level of star formation (SF) because of dust extinction in the disks. The fraction of galaxies considered by Hummel et al. (1991a) as good candidates for follow-up searches of radio halos, based on extended emission found in radio synthesis images, is 10 out of 18 , i.e. $56 \%$.

In previous studies (Dahlem et al. 1995, 1998; hereafter DLG95 and D98, respectively) we have started using a far-infrared criterion, originally defined by Heckman et al. (1990) to characterise starburst galaxies, for selecting galaxies with warm dust as targets for searches of 
gaseous halos: their $60 \mu \mathrm{m}$ to $100 \mu \mathrm{m}$ far-infrared (FIR) IRAS flux ratio. Objects with $f_{60} / f_{100} \gtrsim 0.4$ have warm dust, heated by massive stars, with average dust temperatures, $T_{\mathrm{d}} \gtrsim 35 \mathrm{~K}$. It turns out that all edge-on galaxies selected by us in this way (with $60 \mu \mathrm{m}$ far-infrared [FIR] fluxes of $f_{60} \geq 30 \mathrm{Jy}$ ) show clear signs of halo emission. Emission is detected not only in the radio, but also in other wavebands (D98). Galaxies with known luminous AGNs (that might contribute significantly to the heating of the dust) were excluded from our sample.

Second, contrary to searches of halo gas in very large samples, such as conducted by Hummel et al. (1991a), our present approach of carefully selecting target galaxies is combined with the employment of high-sensitivity observations to detect low surface brightness emission.

Irwin et al. (1999) conducted a search for radio halos in a quite heterogeneous sample of target galaxies. Despite this heterogeneity, these authors achieved a high detection rate of halo emission of $>90 \%$. A discussion of the interpretation of their data in the context of the new results presented here will be provided in Sect. 4.4.

Here we present the results from our radio observations of a number of FIR-warm edge-on galaxies with $3 \mathrm{Jy}<f_{60}<30$ Jy FIR flux densities conducted with the Australia Telescope Compact Array $\left(\mathrm{ATCA}^{1}\right)$ and the Very Large Array $\left(\mathrm{VLA}^{2}\right)$. These galaxies are fainter than the galaxies studied by us previously, because there are too few nearby systems (with $f_{60} \geq 30 \mathrm{Jy}$ ) to derive general properties of their halos. Most objects were selected to be FIR-warm, with $f_{60} / f_{100} \gtrsim 0.4$, except NGC 1055 , NGC 2820 and NGC 7090, which have slightly lower FIR flux ratios. The primary goal is to investigate whether indeed all FIR-warm galaxies (without AGNs) do have gaseous halos and an attempt at detecting these at distances beyond that of the Virgo cluster.

\section{Observations and data reduction}

\subsection{Australia Telescope Compact Array observations}

The galaxies observed by us with the ATCA are NGC 1511, NGC 7090 and NGC 7462. Each was observed with three different configurations of the $750 \mathrm{~m}$ array and two different configurations of the $1.5 \mathrm{~km}$ array, see Table 1 . The individual observing runs were 13 hours long (including time for calibration), providing almost full 12 hour aperture syntheses in each configuration. The total on-source integration times range from $32 \mathrm{~h}$ (NGC 7462) to $47 \mathrm{~h}$ (NGC 1511). The ATCA is capable of observing at two frequencies simultaneously. We observed the radio continuum at $2.45 \mathrm{GHz}$ and $1.43 \mathrm{GHz}$ (13 cm and $21 \mathrm{~cm}$ wavelength, respectively). All data are

1 The Australia Telescope is funded by the Commonwealth of Australia for operation as a National Facility managed by CSIRO.

2 The National Radio Astronomy Observatory (NRAO) is a facility of the National Science Foundation operated under cooperative agreement by Associated Universities, Inc.
Table 1. ATCA observation details.

\begin{tabular}{|c|c|c|c|}
\hline Galaxy & Array $^{a}$ & Date & $\begin{array}{r}\text { Int. time } \\
\text { [h:mm] }\end{array}$ \\
\hline \multirow[t]{6}{*}{ NGC 1511} & $750 \mathrm{~A}$ & 1998, May 03 & 9:00 \\
\hline & $750 \mathrm{~B}$ & 1997, Aug. 10 & $9: 30$ \\
\hline & $750 \mathrm{C}$ & 1997, Oct. 20 & $9: 30$ \\
\hline & $1.5 \mathrm{D}$ & 1998, Oct. 19 & $4: 50$ \\
\hline & $1.5 \mathrm{D}$ & 1998, Oct. 20 & $6: 00$ \\
\hline & $1.5 \mathrm{C}$ & 1999, Apr. 10 & $8: 50$ \\
\hline \multirow[t]{6}{*}{ NGC 7090} & $750 \mathrm{~A}$ & 1998, May 02 & $9: 30$ \\
\hline & $750 \mathrm{~B}$ & 1997, Aug. 06 & $9: 30$ \\
\hline & $750 \mathrm{C}$ & 1997, Oct. 18 & $9: 30$ \\
\hline & $1.5 \mathrm{D}$ & 1998, Oct. 17 & $5: 20$ \\
\hline & $1.5 \mathrm{D}$ & 1998, Oct. 20 & 3:00 \\
\hline & $1.5 \mathrm{~B}$ & 1999, Apr. 02 & $8: 40$ \\
\hline \multirow[t]{5}{*}{ NGC 7462} & $750 \mathrm{~A}$ & 1998, May 04 & 9:00 \\
\hline & $750 \mathrm{~B}$ & 1997, Aug. 08 & $9: 30$ \\
\hline & $750 \mathrm{C}$ & 1997, Oct. 19 & $9: 30$ \\
\hline & $1.5 \mathrm{D}$ & 1998, Oct. 18 & $2: 30$ \\
\hline & $1.5 \mathrm{D}$ & 1998, Oct. 20 & $1: 00$ \\
\hline
\end{tabular}

Note to Table 1:

a) Full aperture synthesis for each ATCA array is obtained by combining data from 4 configurations, named A, B, C and D. Three configurations already provide good $u v$ coverage.

polarisation calibrated. Due to technical problems part of the $1.5 \mathrm{~km}$ array observations of NGC 7462 could not be used.

1934-638 was used as the primary flux calibrator, 0407658 (for NGC 1511) and 2106-413 (for both NGC 7090 and NGC 7462) as polarisation and phase calibrators. The adopted flux of 1934-638 is 11.14 (14.94) Jy at 2.45 (1.43) GHz. The data reduction was performed in a standard fashion, using the software package MIRIAD.

With angular extents of their radio emission of $\lesssim 4^{\prime}$ (see below), the observed galaxies are so small that they fit easily into the primary beam of the ATCA's 22-m antennae (Full Width at Half Maximum $F W H M=20^{\prime} / 34^{\prime}$ at $2.45 / 1.43 \mathrm{GHz}$, respectively) and no primary beam correction is necessary. With a shortest spacing of $45.9 \mathrm{~m}$ and good uv coverage, flux losses due to missing short spacings are negligible.

\subsection{VLA data}

Those galaxies observed with the VLA are listed in Table 2. Two IFs of $50 \mathrm{MHz}$ bandwith were used, centred at $1.465 \mathrm{GHz}$ and $1.385 \mathrm{GHz}$, respectively. Primary flux calibrators are either $0137+331$ (3C 48; $15.62 \mathrm{Jy}$ at $1.465 \mathrm{GHz}$ and $16.32 \mathrm{Jy}$ at $1.385 \mathrm{GHz}$ ) or $1331+305$ (3C $286 ; 14.55 / 14.94 \mathrm{Jy}$, respectively) or both, if available, adopting the flux scale by Baars et al. (1977). The combined final maps have a centre frequency of $1.425 \mathrm{GHz}$. The data reduction was performed in a standard way, utilizing the NRAO software package AIPS. 
Table 2. VLA observation details.

\begin{tabular}{cclc}
\hline Galaxy & Array $^{a}$ & Date & $\begin{array}{c}\text { Int. time } \\
{[\text { h:mm }]}\end{array}$ \\
\hline NGC 1055 & D & 1999, Mar. 25/29 & $3: 30$ \\
NGC 1406 & DnC & 1999, Feb. 16 & $2: 45$ \\
NGC 1421 & D & 1999, Apr. 02 & $2: 45$ \\
NGC 2748 & D & 1999, Apr. 04 & $3: 30$ \\
NGC 2820 & D & 1999, Apr. 04 & $3: 05$ \\
NGC 3175 & DnC & 1999, Feb. 18 & $2: 00$ \\
NGC 3437 & D & 1999, Mar. 29 & $3: 00$ \\
NGC 3717 & DnC & 1999, Feb. 18 & $2: 00$ \\
NGC 4527 & D & 1999, May 28 & $1: 30$ \\
NGC 4700 & D & 1999, May 28 & $1: 35$ \\
NGC 5073 & D & 1999, May 28 & $1: 35$ \\
NGC 7541 & D & 1999, Apr. 02 & $2: 05$ \\
\hline
\end{tabular}

Note to Table 2:

$\left.{ }^{a}\right)$ Southern galaxies $\left(\delta \leq 30^{\circ}\right)$ where observed with a hybrid, i.e., with an extended northern arm of the array.

Due to the shortness of most of the observing runs, no polarisation calibration could be obtained (owing to insufficient parallactic angle coverage).

For galaxies with an extent of $5^{\prime}$ or larger a primary beam correction was performed. This affects NGC 2820 (and its partners), NGC 3717 and NGC 4527. For NGC 3717, one of the most extended objects in our sample, a test was performed on the influence of the primary beam correction on the total flux measurement, which turned out to be negligible ( $0.1 \mathrm{mJy}$ with respect to a total flux of $235 \mathrm{mJy}$ ).

For investigations of the $z$ height of the observed radio emission it is important to have the highest possible resolution in the direction perpendicular to the disk plane of the sample galaxies, while normally interpretation of the results is easiest with a circular beam. In those cases where the clean beam was elongated roughly along the minor axis of the observed galaxy it was restored circular by slightly re-weighting the visibilities. In a few cases, where the beam was elongated in a direction close to the major axis of the target galaxy, it was left elliptical, but restored with its major axis oriented exactly along the galaxy disk. This leaves the beam's minor axis, and thus the highest resolution, perpendicular to the galaxy disk, while keeping the data analysis in the $z$ direction straight-forward.

\section{Results}

\subsection{Radio continuum images}

The 12 galaxies observed with the VLA are displayed in Figs. 1-3, 5-12 and 15. For these objects only $1.425 \mathrm{GHz}$ data are currently available.

ATCA radio continuum images of the three galaxies NGC 1511, NGC 7090 and NGC 7462 at $2.45 \mathrm{GHz}$ and $1.43 \mathrm{GHz}$ are presented in Figs. 4, 13 and 14. Each

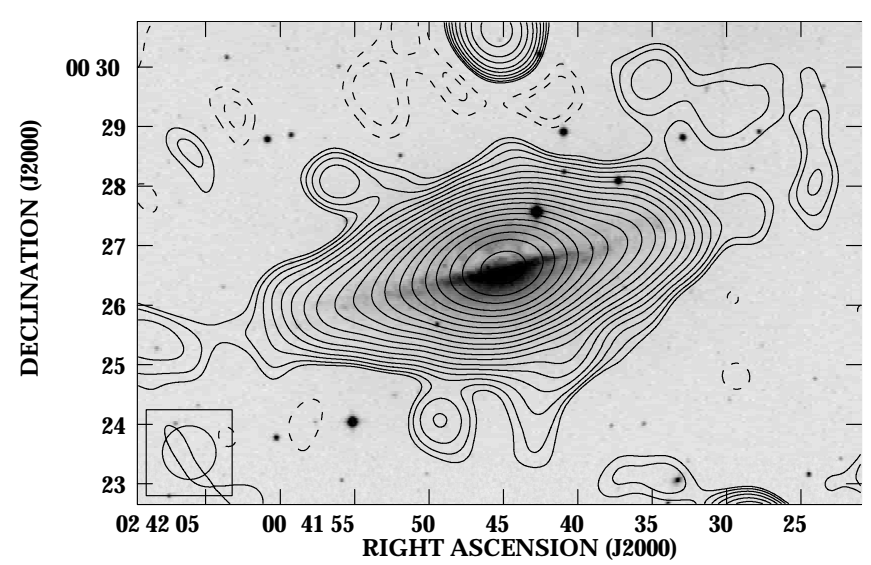

Fig. 1. VLA $1.43 \mathrm{GHz}$ radio continuum contour map of NGC 1055, superimposed on a Digital Sky Survey (DSS) image. The contour levels displayed are $-0.23,-0.16,0.16(=2 \sigma)$, $0.23,0.32,0.45, \ldots, 80.2$ mJy beam $^{-1}$, with a contour spacing of a factor of $\sqrt{2}$.

figure displays the $2.45 \mathrm{GHz}$ image on the left side and the $1.43 \mathrm{GHz}$ map on the right.

In the following we briefly describe the radio images of each galaxy in our small sample of 15 systems. Note that the first positive contour always displays the $2 \sigma$ confidence level and further contours increase by factors of $\sqrt{2}$ from there.

The angular resolution and sensitivities $(1 \sigma)$ of the maps are listed in Table 3 . We also include in Table 3 the measured total $1.43 \mathrm{GHz}$ flux densities, $S_{1.43}$, and the position angles of the radio continuum emission distribution, as determined from fitting two-dimensional ellipsoidal Gaussians to the radio continuum emission distributions, using the AIPS task JMFIT.

NGC 1055: The $1.425 \mathrm{GHz}$ radio continuum emission of NGC 1055 is displayed in Fig. 1. It is centrally peaked, with resolved disk emission out to radii of about 4.5 . As in the subsequent figures, the full width at half maximum ( $F W H M)$ of the beam is displayed in the lower left corner. There are several nearby point sources, which are most likely unrelated.

NGC 1406: Like NGC 1055, NGC 1406 (Fig. 2) exhibits centrally peaked emission from its disk, however with little substructure at the angular resolution of the current data.

NGC 1421: NGC 1421 is resolved by our observations, which were combined with data by Irwin et al. (1999). The emission is centrally peaked, but the central maximum is not very bright (Fig. 3). Several nearby unrelated point sources had to be removed for investigations of its structure perpendicular to the disk plane. 
Table 3. Basic properties and imaging parameters.

\begin{tabular}{ccccccccrr}
\hline Galaxy & $\begin{array}{c}\alpha \\
(2000)\end{array}$ & $\begin{array}{c}\delta \\
(2000)\end{array}$ & $\begin{array}{c}D \\
(\mathrm{Mpc})\end{array}$ & $\begin{array}{c}i^{a} \\
\left(^{\circ}\right)\end{array}$ & $\begin{array}{c}f_{60} \\
f_{100}\end{array}$ & $\begin{array}{c}\text { Beam }^{b} \\
F W H M\left({ }^{\prime \prime}\right)\end{array}$ & $\begin{array}{c}1 \sigma \mathrm{rms} \\
\left(\mathrm{mJy} \mathrm{beam}^{-1}\right)\end{array}$ & $\begin{array}{c}S_{1.43} \\
(\mathrm{mJy})\end{array}$ & $\begin{array}{c}\text { Pos. Angle } \\
\text { PA }\left(^{\circ}\right)\end{array}$ \\
\hline NGC 1055 & 024143.7 & +002554 & 16.0 & 67 & 0.34 & 54 & 0.08 & $233 \pm 10$ & $102.6 \pm 0.2$ \\
NGC 1406 & 033922.5 & -311919 & 14.9 & 90 & 0.44 & 43 & 0.08 & $127 \pm 7$ & $16.2 \pm 0.3$ \\
NGC 1421 & 034229.5 & -132923 & 31.1 & 90 & 0.40 & $57 \times 37.5(30)$ & 0.10 & $110 \pm 7$ & $0.5 \pm 0.1$ \\
NGC 1511 & 035935.7 & -673807 & 17.5 & 72 & 0.62 & $17.7^{c} / 33.0^{d}$ & $0.070^{c} / 0.05^{d}$ & $156 \pm 8$ & $122.8 \pm 0.2$ \\
NGC 2748 & 091344.6 & +762832 & 28.7 & 71 & 0.39 & 52 & 0.10 & $66.4 \pm 6$ & $42.1 \pm 0.5$ \\
NGC 2820 & 092144.1 & +641526 & 29.9 & 90 & $0.33^{e}$ & $62 \times 41(75)$ & 0.06 & $59.0 \pm 8$ & $67.4 \pm 0.2$ \\
NGC 3175 & 101442.3 & -285220 & 15.9 & 85 & 0.46 & $43 \times 33(52)$ & 0.07 & $69.9 \pm 6$ & $51.2 \pm 0.2$ \\
NGC 3437 & 105234.9 & +225604 & 25.5 & 75 & 0.57 & $70 \times 41(-71)$ & 0.10 & $73.6 \pm 6$ & $111.6 \pm 0.1$ \\
NGC 3717 & 113131.8 & -301832 & 27.1 & 90 & 0.44 & 41 & 0.08 & $123 \pm 10$ & $33.7 \pm 0.6$ \\
NGC 4527 & 123408.5 & +023911 & 31.9 & 68 & 0.47 & $55 \times 50(67)$ & 0.15 & $235 \pm 10$ & $68.3 \pm 0.2$ \\
NGC 4700 & 124907.3 & -112446 & 25.5 & 90 & 0.57 & $67 \times 46(50)$ & 0.10 & $29.6 \pm 3$ & $49.8 \pm 0.8$ \\
NGC 5073 & 131920.8 & -145035 & 43.9 & 87 & 0.69 & $71 \times 44(0)$ & 0.15 & $38.9 \pm 4$ & $176.4^{f} \pm 0.4$ \\
NGC 7090 & 213628.6 & -543326 & 11.7 & 90 & 0.33 & $17.7^{c} / 34.5^{d}$ & $0.075^{c} / 0.05^{d}$ & $38.0 \pm 4$ & $128.2 \pm 0.2$ \\
NGC 7462 & 230246.5 & -405007 & 15.1 & 90 & 0.49 & $23.5^{c} / 46.5^{d}$ & $0.085^{c} / 0.05^{d}$ & $26.0 \pm 3$ & $69.7 \pm 1.0$ \\
NGC 7541 & 231443.0 & +043205 & 42.4 & 75 & 0.48 & 47 & 0.15 & $158 \pm 8$ & $95.6 \pm 0.4$ \\
\hline
\end{tabular}

Notes to Table 3:

$\left.{ }^{a}\right)$ Inclination angle; approximate values based on optical axial ratios, from Tully (1988). In particular the value for NGC 1055 appears to be too low, possibly because of its prominent bulge.

$\left.{ }^{b}\right)$ Major axis $\times$ minor axis (position angle), if not circular.

c) At $2.45 \mathrm{GHz}$ observing frequency.

$\left.{ }^{d}\right)$ At $1.43 \mathrm{GHz}$ observing frequency.

$\left.{ }^{e}\right)$ The FIR flux measurements of NGC 2820 are contaminated by contributions from its partners, NGC 2820 A and NGC 2814 .

$\left.{ }^{f}\right)$ NGC 5073 is basically unresolved; the radio $P A$ reflects the beam shape. During the data analysis $\mathrm{PA}=56^{\circ}$ was used.

NGC 1511: NGC 1511 (Fig. 4) has an unusual radio continuum distribution in its disk. There are two prominent maxima, about $30^{\prime \prime}$ apart. The surface brightness of the emission is high at both $2.45 \mathrm{GHz}$ and $1.43 \mathrm{GHz}$. Perpendicular to the disk plane the emission distribution is very thick. The total height of the emission distribution at $2.45 \mathrm{GHz}$ is about 5 beamwidths (about 4 at $1.43 \mathrm{GHz}$ ). The axial ratio in the optical is 2.9 (NASA Extragalactic Database; NED), while the radio axial ratio is approximately 1.4 (at $2.45 \mathrm{GHz}$ ) and even 1.2 at $1.43 \mathrm{GHz}$.

None of the radio continuum emission from NGC 1511 is associated with SN 1935C (or "Nova" Hyi 1935; van den Bergh \& Hazen 1988), which occurred further to the north-east. Also the two sources about $2^{\prime}$ south of NGC 1511 are probably unrelated.

NGC 2748: The radio continuum emission distribution of NGC 2748, displayed in Fig. 5, is centrally peaked but shows almost no significant substructure at the current resolution. The source to the west of NGC 2748 is unrelated.

NGC 2820: Figure 6 shows a complex emission distribution, comprised of contributions from NGC 2820 (east of the centre) and NGC 2814 (to the west) as the major peaks and NGC 2820A as a tertiary maximum in between these two. Fitting Gaussians to the emission peaks of these sources, a fourth component remains that is associated with the companion of NGC 2814. Another member of this galaxy group, NGC 2805 (outside the displayed field of view) was also detected.

This proximity of the different emission sources makes it difficult to disentangle the contributions from each of the systems. Using Gaussian ellipsoids to fit the individual emission distributions, we succeeded in separating the radio emitters. However, the same problem of flux contamination also applies to the FIR data, based on which the target was selected (see Table 4), where the sources cannot be spatially separated due to the lower angular resolution of IRAS compared with our data.

NGC 3175: The radial extent of NGC 3175's radio continuum emission distribution (Fig. 7) is small compared to its optical extent (Condon et al. 1996). The emission is centrally peaked and marginally resolved along the disk plane, with no directly visible substructure. The source to the north-west is unrelated.

NGC 3437: NGC 3437 (Fig. 8) shows little substructure in its radio continuum emission distribution at the present resolution.

NGC 3717: The radio continuum emission distribution of NGC 3717 is complex (Fig. 9). Besides a strong central peak extended disk emission is visible as an emission ridge 


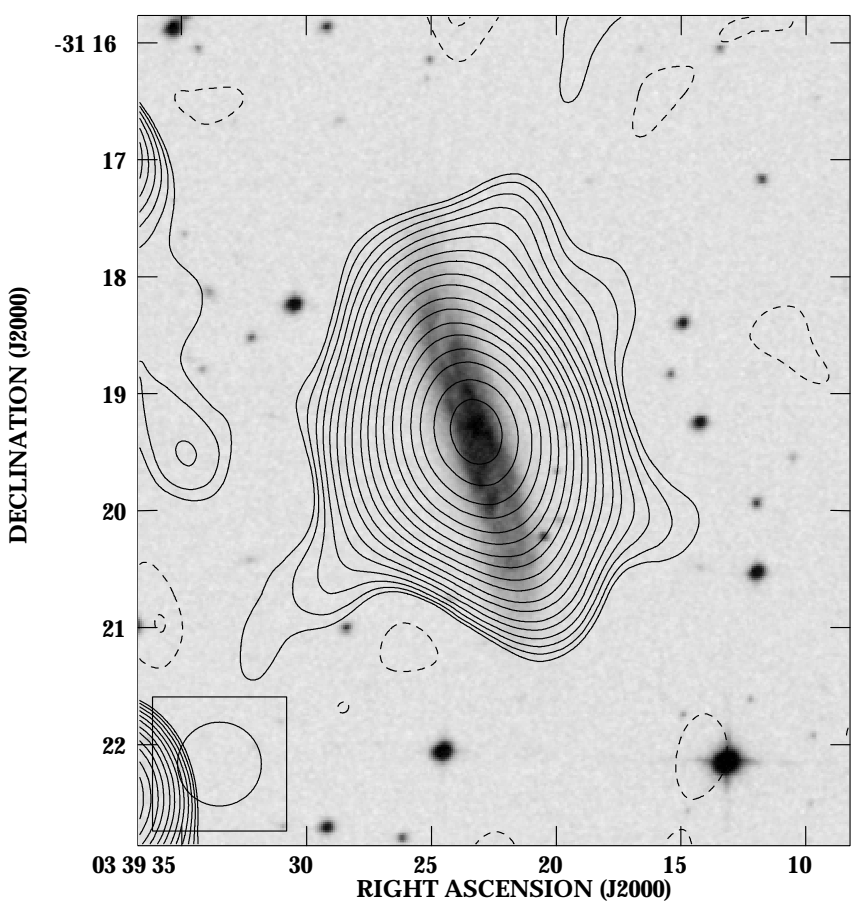

Fig. 2. VLA $1.43 \mathrm{GHz}$ radio continuum contour map of NGC 1406, superimposed on a Digital Sky Survey (DSS) image. The contour levels displayed are $-0.23,-0.16,0.16(=2 \sigma)$, $0.23,0.32,0.45, \ldots, 40.1$ mJy beam $^{-1}$, with a contour spacing of a factor of $\sqrt{2}$.

along the major axis, with a secondary emission peak about $3^{\prime}$ south-west of the centre and another, weaker source about $3^{\prime}$ north-east of the nucleus. The total radial extent of the emission distribution is about $7^{\prime}$. South-east of the centre, further emission is visible away from the disk plane, which is not easily removable by subtracting a point source profile and might therefore be genuinely extended. The weak point source north-west of the centre is probably unrelated.

NGC 4527: The radio continuum emission of NGC 4527 , as displayed in Fig. 10, is bright, centrally peaked and extended along its major axis, with an extent of about 6.5 . The ratio of major-to-minor axis extent is less than 2 , which is low for a highly inclined galaxy. The fact that the minor axis extent of the emission distribution is about 4 beamwidths indicates that it is resolved in this direction, despite the fact that there is little visible substructure. The central source, which is classified as an H II/LINER nucleus (NED), was subtracted for further processing (see Sect. 3.2).

NGC 4700: Despite its small angular extent, the radio continuum emission distribution of NGC 4700 (Fig. 11) is resolved in both the major and minor axis direction. The axial ratio of the emission distribution is only 1.25 , which is extremely low. This is a clear indication of extraplanar emission, especially when the elliptical shape of the

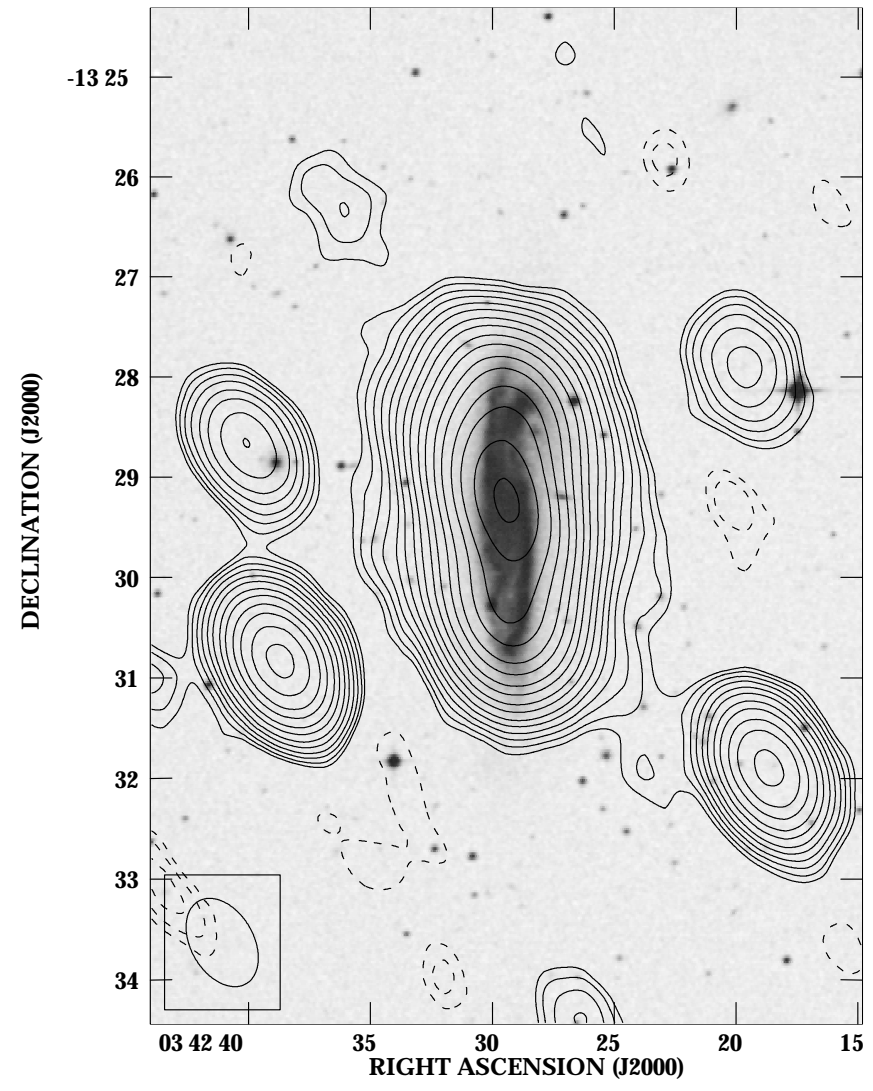

Fig. 3. VLA C+D array $1.465 \mathrm{GHz}$ radio continuum contour map of NGC 1421, superimposed on a Digital Sky Survey (DSS) image. The contour levels displayed are $-0.4,-0.28$, $-0.2,0.2(=2 \sigma), 0.28,0.44,0.56,0.8, \ldots, 35.2 \mathrm{mJy} \mathrm{beam}^{-1}$, with a contour spacing of a factor of $\sqrt{2}$.

beam, with its major axis aligned with that of the galaxy, is taken into account.

Two strong point sources east of NGC 4700 (one just outside the displayed field of view) are unrelated. A third, weaker point source about 2.5 south-west of the galaxy centre might possibly be related with NGC 4700, being located near its disk plane, however beyond the radial limit of the optical emission distribution. This point source was not included in the total flux measurement and when determining the position angle, PA (Table 3 ).

NGC 5073: The emission of NGC5073 is unresolved (Fig. 12), with no immediately visible sign of extraplanar emission at the angular resolution of the current data.

NGC 7090: NGC 7090 (Fig. 13) exhibits centrally peaked radio continuum emission from its disk. The radial extent of the disk emission is about $4^{\prime}$ at $2.45 \mathrm{GHz}$ and 5.5 at $1.43 \mathrm{GHz}$. The disk emission is clearly resolved, with secondary maxima most clearly visible in the $2.45 \mathrm{GHz}$ map.

There are also clear indications of extraplanar emission from cosmic ray electrons (CRs) in the halo. The halo emission is detected and spatially resolved at both 

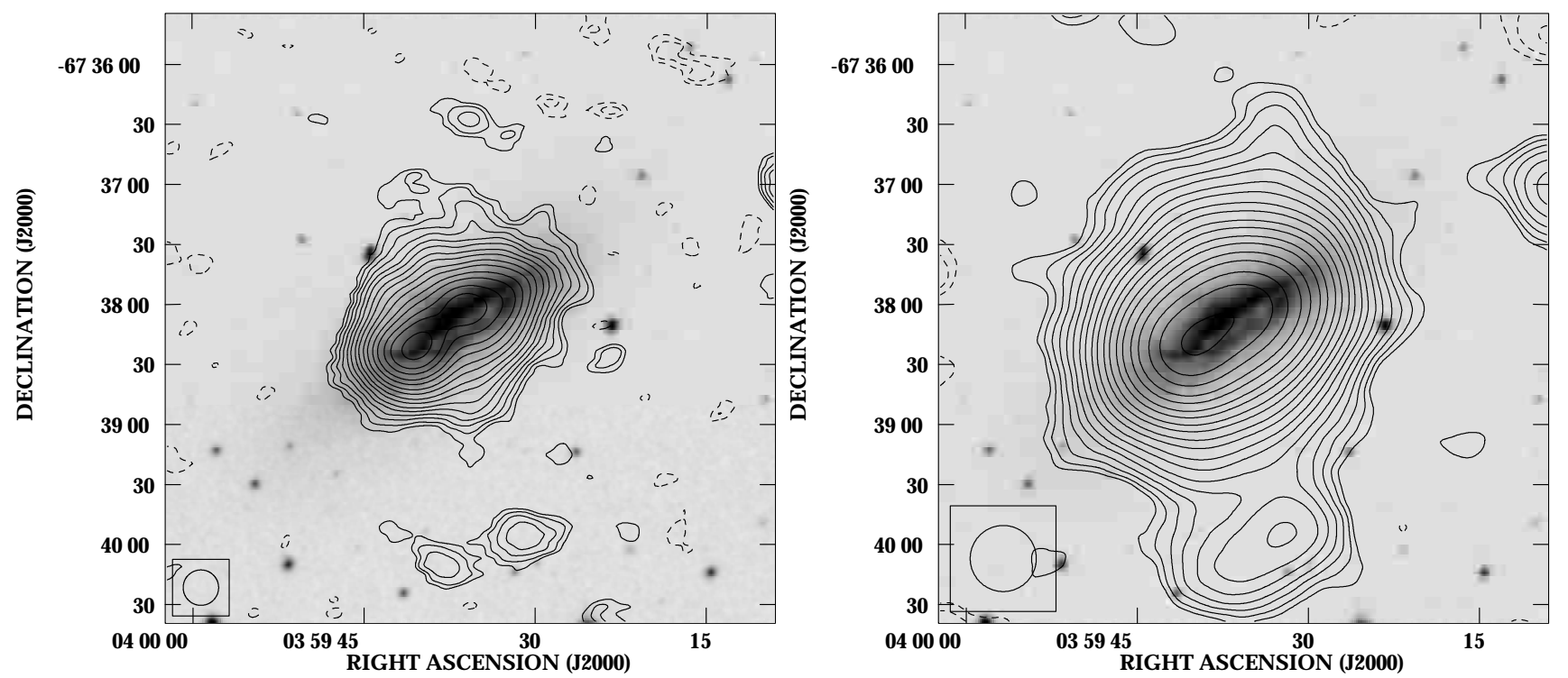

Fig. 4. ATCA radio continuum maps of NGC 1511. Left panel: $2.45 \mathrm{GHz}$ map; right panel: $1.43 \mathrm{GHz}$ map; both are overlaid on a DSS optical image. The contours display the $-4,-2.8,-2,2,2.8,4, \ldots \sigma$ confidence level (the $1-\sigma \mathrm{rms}$ is $0.07 / 0.05 \mathrm{mJy}$ beam $^{-1}$ at $2.45 / 1.43 \mathrm{GHz}$, respectively).

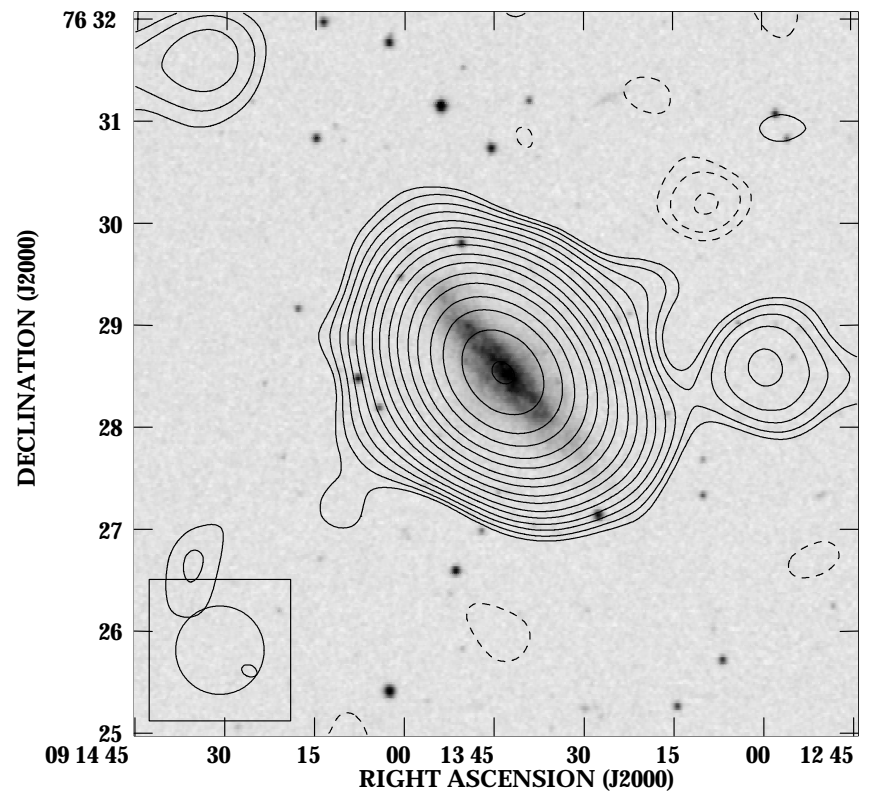

Fig. 5. VLA $1.43 \mathrm{GHz}$ radio continuum contour map of NGC 2748, superimposed on a Digital Sky Survey (DSS) image. The contour levels displayed are $-0.4,-0.28,-0.2,0.2$ $(=2 \sigma), 0.28,0.44,0.56,0.8, \ldots, 35.2$ mJy beam $^{-1}$, with a contour spacing of a factor of $\sqrt{2}$.

observing frequencies, $2.45 \mathrm{GHz}$ and $1.43 \mathrm{GHz}$. The $1.43 \mathrm{GHz}$ halo emission is apparently more extended and clearly brighter than at $2.45 \mathrm{GHz}$.

NGC 7462: Figure 14 displays centrally peaked emission in the disk of NGC 7462 at both $2.45 \mathrm{GHz}$ and $1.43 \mathrm{GHz}$. The $2.45 \mathrm{GHz}$ map exhibits a secondary emission maximum about 1.3 east of the centre and a weaker corresponding maximum to the west of the nucleus.

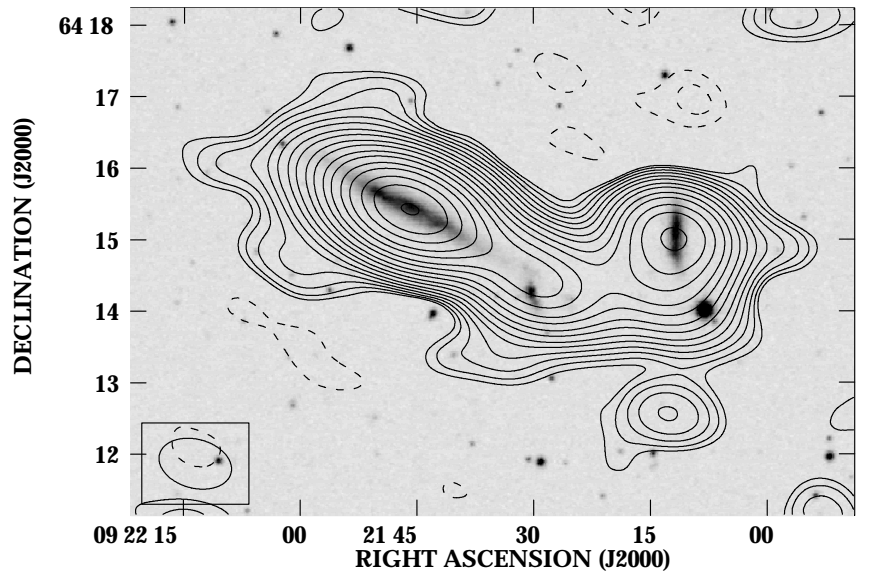

Fig. 6. VLA $1.43 \mathrm{GHz}$ radio continuum contour map of NGC 2820, superimposed on a Digital Sky Survey (DSS) image. The contour levels displayed are $-0.17,-0.12,0.17$, $0.24, \ldots, 30.7 \mathrm{mJy}^{\text {beam }}{ }^{-1}$, with a contour spacing of a factor of $\sqrt{2}$.

The $2.45 \mathrm{GHz}$ map also shows some emission south of the centre, outside the disk of NGC 7462. At $1.43 \mathrm{GHz}$ one can discern extended emission from the halo. Despite the different angular resolutions of the two maps it appears that the halo is more prominent at $1.43 \mathrm{GHz}$ than at higher frequencies. The $2.45 \mathrm{GHz}$ map may also lack sensitivity and thus not show the full extent of the emission at this frequency yet. Note that the total flux densities of this galaxy at $2.45 \mathrm{GHz}$ and $1.43 \mathrm{GHz}$ are quite low (Table 3).

NGC 7541: NGC7541 (Fig. 15) was observed in bad weather conditions, which caused phase problems that could not be removed entirely from the visibility data 
Table 4. Measurements of the exponential disk and halo $z$ scale heights.

\begin{tabular}{|c|c|c|c|c|c|c|c|c|c|}
\hline \multirow[t]{2}{*}{ Galaxy } & \multirow{2}{*}{$\begin{array}{l}\text { Obs. } \\
\text { Freq. } \\
(\mathrm{GHz})\end{array}$} & \multirow{2}{*}{$\begin{array}{c}\text { Radial }^{a} \\
\text { Range } \\
\left({ }^{\prime \prime} / \mathrm{kpc}\right)\end{array}$} & \multirow{2}{*}{$\begin{array}{c}\text { Resolution } \\
\perp \text { Major Axis } \\
\left({ }^{\prime \prime} / \mathrm{kpc}\right)\end{array}$} & \multicolumn{2}{|c|}{ Disk } & \multicolumn{4}{|c|}{ Halo } \\
\hline & & & & $\begin{array}{c}z_{0, \text { disk }} \\
\left({ }^{\prime \prime} / \mathrm{kpc}\right)\end{array}$ & $\begin{array}{l}\text { Rel. } \\
\text { Ampl. }\end{array}$ & Side $1^{b}$ & $\begin{array}{l}\text { halo }\left({ }^{\prime \prime} / \mathrm{kpc}\right) \\
\text { Side } 2^{b}\end{array}$ & Average & $\begin{array}{l}\text { Rel. } \\
\text { Ampl. }\end{array}$ \\
\hline NGC 1055 & 1.425 & $\pm 150 / \pm 11.6$ & $54.0 / 4.19$ & $19.2 / 1.49$ & 1.00 & - & - & - & 0.00 \\
\hline NGC 1406 & 1.425 & $\pm 66 / \pm 4.8$ & $43.0 / 5.28$ & $4.8 / 0.35$ & 0.85 & $27.3 / 1.97$ & $18.6 / 1.34$ & $23.0 / 1.66$ & 0.15 \\
\hline NGC 1421 & 1.465 & $\pm 90 / \pm 13.6$ & $37.5 / 5.66$ & $13.3 / 2.01$ & 1.00 & - & - & - & 0.00 \\
\hline \multirow{2}{*}{ NGC 1511} & 1.43 & $\pm 34 / \pm 2.9$ & $33.0 / 2.80$ & $10.5 / 0.89$ & 0.96 & $14.9 / 1.27$ & $26.6 / 2.26$ & $20.7 / 1.76$ & 0.04 \\
\hline & 2.45 & $\pm 60 / \pm 5.1$ & $17.7 / 1.50$ & $7.9 / 0.67$ & 0.90 & $17.9 / 1.52$ & $18.1 / 1.54$ & $18.0 / 1.53$ & 0.10 \\
\hline NGC 2748 & 1.425 & $\pm 78 / \pm 10.9$ & $52.0 / 7.24$ & $12.5 / 1.74$ & 1.00 & - & - & - & 0.00 \\
\hline NGC 2820 & 1.425 & $\pm 42 / \pm 6.1$ & $41.0 / 5.95$ & $10.8 / 1.57$ & 1.00 & - & - & - & 0.00 \\
\hline \multirow[t]{2}{*}{ NGC 3175} & 1.425 & $\pm 54 / \pm 4.2$ & $33.0 / 2.55$ & $10.3 / 0.80$ & 0.94 & $20.3 / 1.57$ & $0.0 / 0.0$ & $10.2 / 0.79$ & 0.06 \\
\hline & $1.49^{c}$ & $\pm 46 / \pm 3.5$ & $15.0 / 1.16$ & $5.3 / 0.41$ & 0.84 & $27.6 / 2.13$ & $14.4 / 1.11$ & $21.0 / 1.62$ & 0.16 \\
\hline NGC 3437 & 1.425 & $\pm 66 / \pm 8.2$ & $41.0 / 5.07$ & $9.8 / 1.21$ & 1.00 & - & - & - & 0.00 \\
\hline NGC 3717 & 1.425 & $\pm 90 / \pm 11.8$ & $41.0 / 5.39$ & $14.2 / 1.87$ & 1.00 & - & - & - & 0.00 \\
\hline NGC 4527 & 1.425 & $\pm 114 / \pm 17.6$ & $50.0 / 7.74$ & $20.8 / 3.22$ & 1.00 & - & - & - & 0.00 \\
\hline NGC 4700 & 1.425 & $\pm 78 / \pm 9.7$ & $46.0 / 5.69$ & $0.1 / 0.01$ & 0.33 & $26.9 / 3.33$ & $22.5 / 2.78$ & $24.7 / 3.06$ & 0.67 \\
\hline NGC 5073 & 1.425 & $\pm 66 / \pm 14.1$ & $44.0 / 9.37$ & $14.7 / 3.13$ & 1.00 & - & - & - & 0.00 \\
\hline \multirow[t]{2}{*}{ NGC 7090} & 1.43 & $\pm 74 / \pm 4.2$ & $34.5 / 1.96$ & $7.0 / 0.40$ & 0.58 & $24.5 / 1.39$ & $38.0 / 2.16$ & $31.2 / 1.77$ & 0.42 \\
\hline & 2.45 & $\pm 124 / \pm 7.0$ & $17.7 / 1.00$ & $2.5 / 0.14$ & 0.59 & $21.0 / 1.19$ & $29.0 / 1.65$ & $25.0 / 1.42$ & 0.41 \\
\hline \multirow[t]{2}{*}{ NGC 7462} & 1.43 & $\pm 54 / \pm 4.0$ & $46.5 / 3.41$ & $10.0 / 0.73$ & 0.65 & $25.3 / 1.85$ & $24.4 / 1.79$ & $24.8 / 1.82$ & 0.35 \\
\hline & 2.45 & $\pm 76 / \pm 5.6$ & $23.5 / 1.72$ & $2.5 / 0.18$ & 0.66 & $26.2 / 1.92$ & $20.6 / 1.51$ & $23.4 / 1.71$ & 0.34 \\
\hline NGC 7541 & 1.425 & $\pm 54 / \pm 11.1$ & $47.0 / 9.67$ & $9.8 / 2.02$ & 1.00 & - & - & - & 0.00 \\
\hline
\end{tabular}

Notes to Table 4:

$\left.{ }^{a}\right)$ Range (in units of arcsec and $\mathrm{kpc}$ ) parallel to the galaxy's major axis over which data were averaged.

b) "Side 1" is always the left-hand side in the plots in Figs. 18-33, "Side 2" the right-hand side.

$\left.{ }^{c}\right)$ Data from Condon et al. (1996).

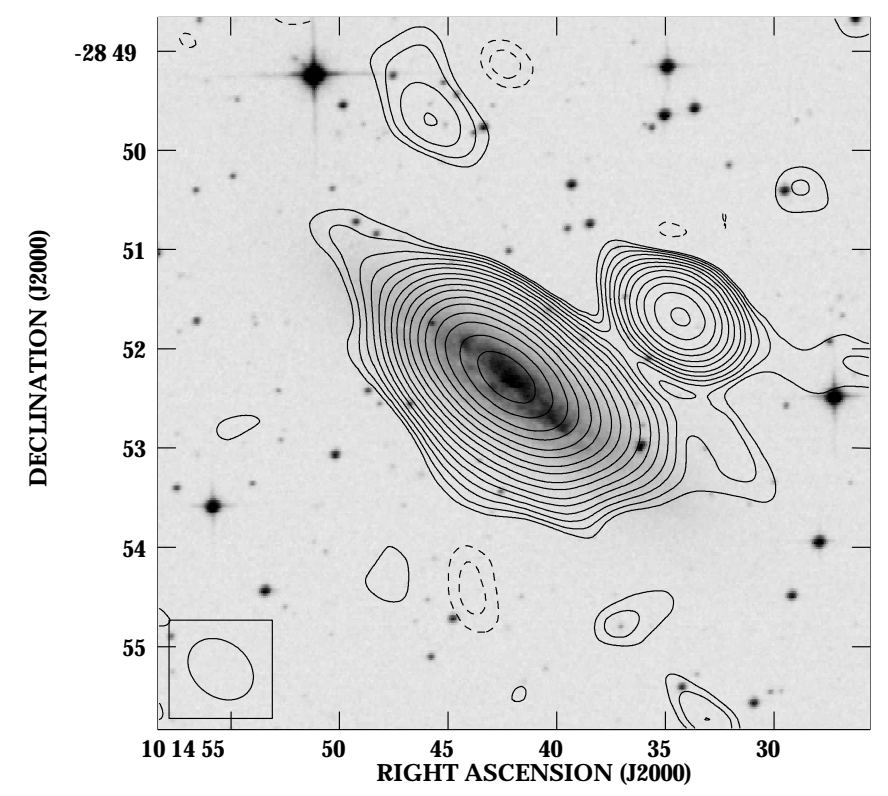

Fig. 7. VLA $1.43 \mathrm{GHz}$ radio continuum contour map of NGC 3175, superimposed on a Digital Sky Survey (DSS) image. The contour levels displayed are $-0.2,-0.14,0.14(=2 \sigma)$, $0.2,0.28,0.4, \ldots, 24.64 \mathrm{mJy}$ beam $^{-1}$, with a contour spacing of a factor of $\sqrt{2}$.

using the self-calibration technique. The $1.43 \mathrm{GHz}$ emission distribution is centrally peaked and marginally resolved along the major axis, without visible substructure

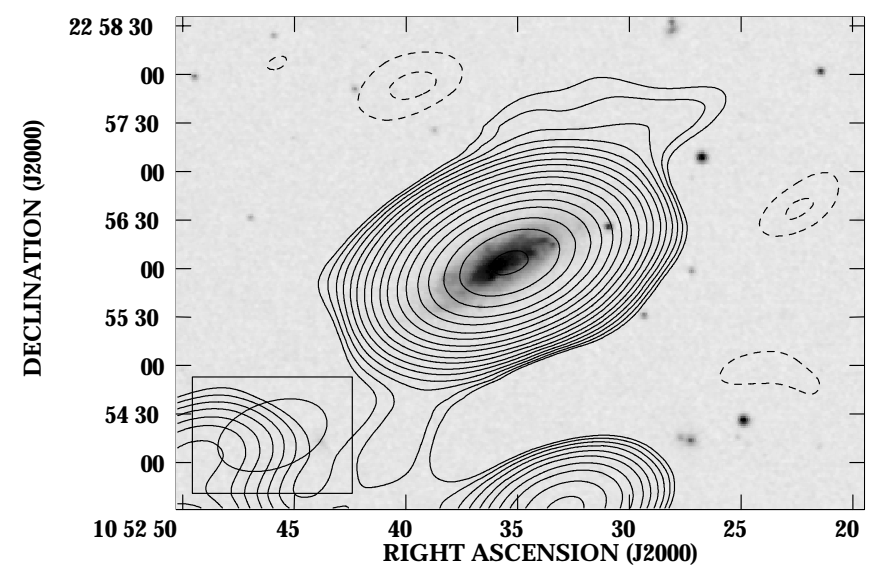

Fig. 8. VLA $1.43 \mathrm{GHz}$ radio continuum contour map of NGC 3437, superimposed on a Digital Sky Survey (DSS) image. The contour levels displayed are $-0.28,-0.2,0.2(=2 \sigma), 0.28$, $0.44,0.56,0.8, \ldots, 35.2 \mathrm{mJy}^{\text {beam }^{-1}}$, with a contour spacing of a factor of $\sqrt{2}$.

at the current resolution. The source to the south-west of NGC 7541 is NGC 7537. The other nearby point sources are probably also unrelated.

\subsection{Investigations of the $z$ structure}

In order to investigate the $z$ structure of the sample galaxies (i.e., the emission distribution perpendicular to the 


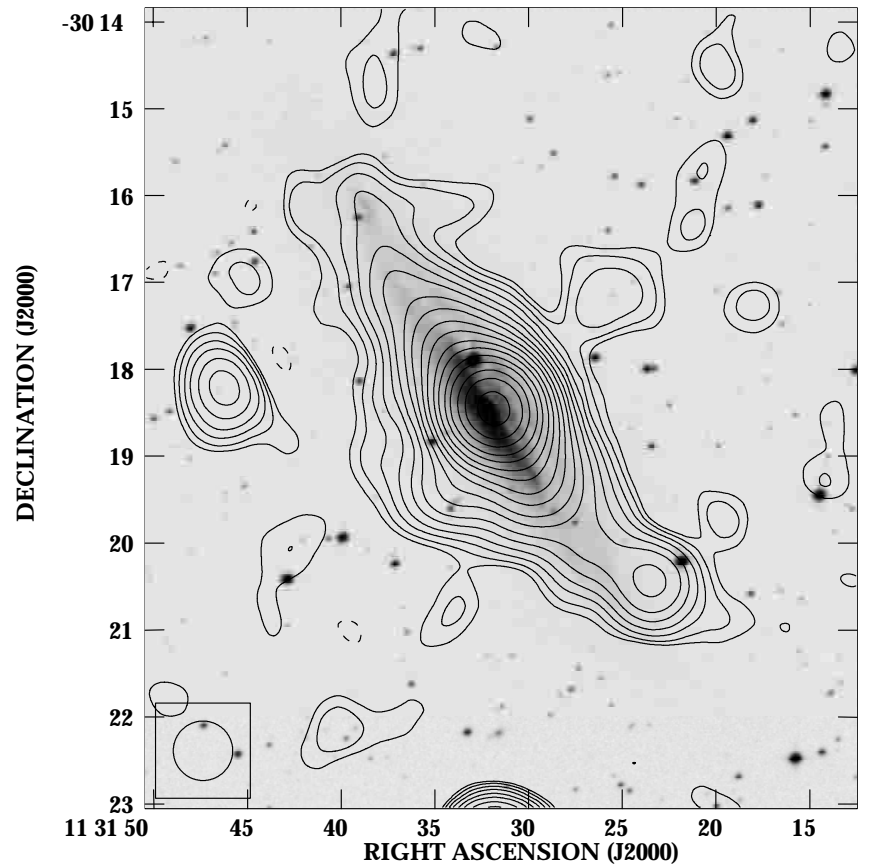

Fig. 9. VLA $1.43 \mathrm{GHz}$ radio continuum contour map of NGC 3717, superimposed on a Digital Sky Survey (DSS) image. The contour levels displayed are $-0.16,0.16(=2 \sigma), 0.23$, $0.32,0.45, \ldots, 56.7 \mathrm{mJy}^{\text {beam }^{-1}}$, with a contour spacing of a factor of $\sqrt{2}$.

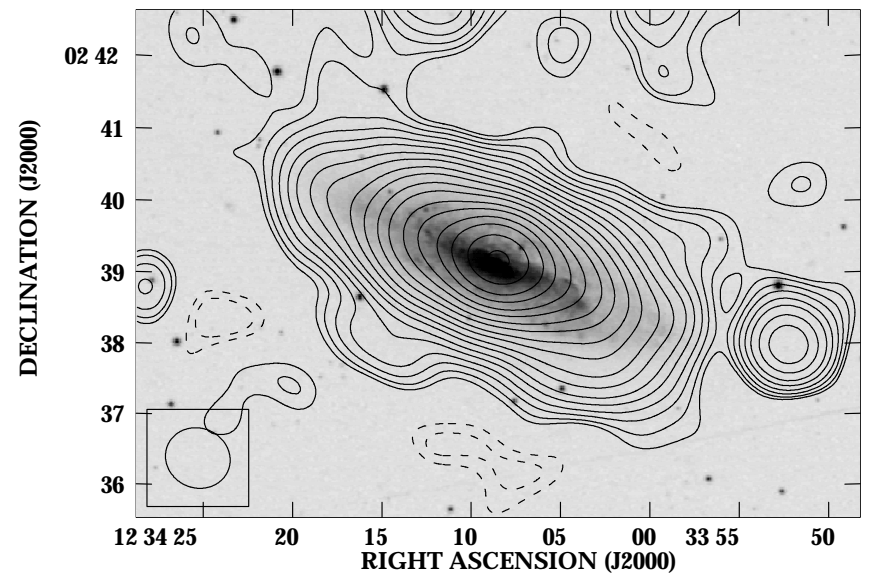

Fig. 10. VLA $1.43 \mathrm{GHz}$ radio continuum contour map of NGC 4527, superimposed on a Digital Sky Survey (DSS) image. The contour levels displayed are $-0.42,-0.3,0.3(=2 \sigma)$, $0.42,0.6,0.84, \ldots, 105.6 \mathrm{mJy}^{\text {beam }^{-1}}$, with a contour spacing of a factor of $\sqrt{2}$.

disk plane), the following technique was applied (as done by us earlier; see e.g. Hummel et al. 1991b; Dahlem et al. 1994; Dahlem et al. 1997):

1. First, nearby confusing point sources were removed, using the AIPS routine JMFIT for two-dimensional ellipsoidal Gaussian fitting of the source profiles;

2 . then the unresolved nuclear source was fitted and removed from those galaxies where the nuclear emission contributes $\geq 25 \%$ of the total flux density. This applies to NGC 1055, NGC 1406, NGC 3717 and NGC 4527;
3. Subsequently the radial range was defined over which radio data were averaged and then an average cut through the galaxy, parallel to its minor axis, was produced over that radial range (called a " $z$ profile");

4. The peak surface brightness in this $z$ profile was normalised to unity and the resulting graph displayed as a solid line in the plots introduced below;

5. A cut through a Gaussian representing the shape of the restored beam along the galaxy's minor axis was created and its peak also normalised to unity. This Gaussian (the beam shape) represents the instrumental response to an infinitesimally thin disk at perfect edge-on inclination of $90^{\circ}$. It is shown in all plots of $z$ profiles as a bold line;

6. An exponential function was created, modelling the $z$ structure of a (thin) disk, which was also normalised in its maximum to unity. The width of this function may represent the intrinsic thickness of the disk, widening of the emission distribution of an infinitely thin disk due to non-perfect edge-on viewing geometry, or a superposition of both;

7. This exponential was then convolved with the beam and the width of the resulting distribution adjusted to mimic the width of the central maximum in the observed $z$ profile;

8. In case this results in a good fit to the data, no further processing is required and it is assumed that no halo emission was detected;

9. In those cases where residual emission, in particular "wings" in the $z$ profiles, cannot be fitted by a single beam-convolved exponential function, a second, wider beam-convolved exponential was added to the model. This second component mimics the behaviour of that part of the emission distribution that we associate with a radio halo;

10. Due to asymmetries in the observed emission distributions, the exponential scale heights of this wide emission component were allowed to be different on either side of the disk plane, with a common maximum in the disk plane to ensure continuity of the model distribution;

11. This two-component model was then fitted to the data. The relative amplitudes of both components are free fit parameters, with the constraint that the sum of both amplitudes be 1;

12. The following profiles were then plotted together for each galaxy, on a logarithmic scale:

(a) The observed $z$ profile (solid line),

(b) the restored beam (bold line),

(c) the beam-smeared exponential thin disk model (dotted line),

(d) the beam-smeared exponential halo model (second dotted line; only if required by fit), and

(e) the sum of both components (i.e. the "best fit" model to the data; dashed line).

The relevant parameters and results of this procedure are collected in Table 4. The graphs described above for 


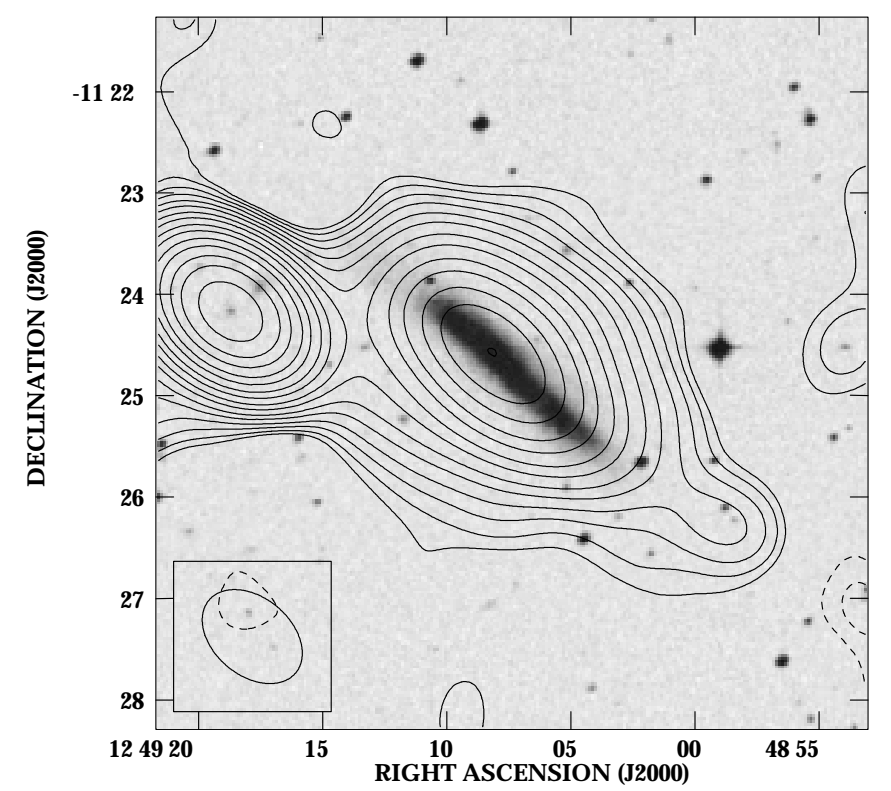

Fig. 11. VLA $1.43 \mathrm{GHz}$ radio continuum contour map of NGC 4700, superimposed on a Digital Sky Survey (DSS) image. The contour levels displayed are $-0.28,-0.2,0.2(=2 \sigma), 0.28$, $0.44,0.56,0.8, \ldots, 35.2$ mJy beam $^{-1}$, with a contour spacing of a factor of $\sqrt{2}$.

each of the 15 galaxies are reproduced in Figs. 18-33. The $1.43 \mathrm{GHz}$ data of NGC 7090 serve as a showcase to demonstrate the above steps.

NGC 7090: The relatively high resolution of our data of NGC 7090 allows for a clear distinction between disk and halo emission in this galaxy. Therefore, we use it here as a showcase to demonstrate the process of modeling the $z$ distribution of the emission. The observed emission distribution is reproduced on a linear scale as the solid line in the left panel of Fig. 16. An exponential disk model was created (narrow peaked distribution, solid line) and convolved with the beam (bold line), leading to the smoother profile (dotted line). Its amplitude is initially fixed at 1 to see whether it can fit the observed emission distribution. However, deviations from the disk model set in at about $25-30 \%$ of the peak surface brightness. In such cases, when residuals remain, a second, wider exponential is added and beam-convolved. The two components are then summed and the resulting function (dashed line) fitted to the data. For easier inspection of the exponential wings, the data and model, including its individual components, are then reproduced on a logarithmic scale (right panel of Fig. 16). One can see that the halo emission is asymmetric, with brighter emission towards positive offsets (the north-east) than south-west of the disk. To save space, only logarithmic plots are shown for all other galaxies. Deviations from a purely exponential decay are visible at $2.45 \mathrm{GHz}$ (Fig. 17). These might be caused by the spurs with relatively high surface brightness detected by Harnett \& Reynolds (1985). Such details are visible, because the $2.45 \mathrm{GHz}$ map of NGC 7090 is not only one of two maps

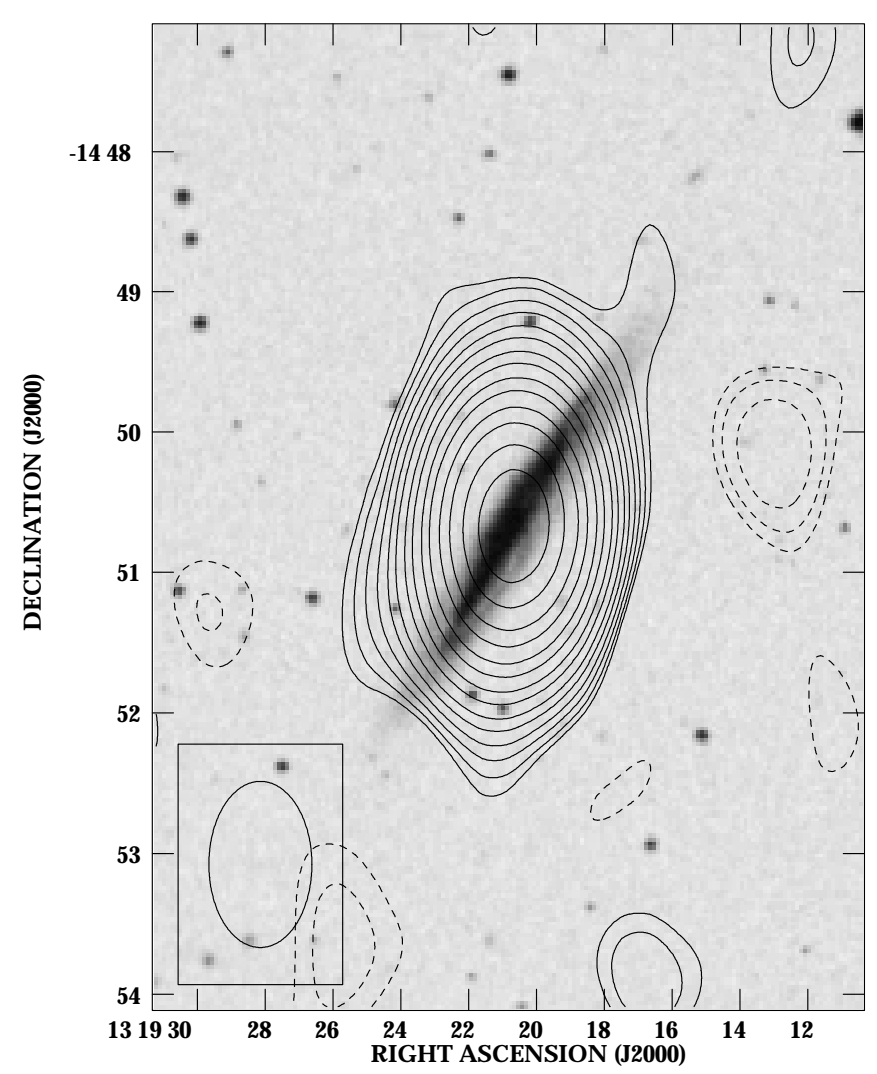

Fig. 12. VLA $1.43 \mathrm{GHz}$ radio continuum contour map of NGC 5073, superimposed on a Digital Sky Survey (DSS) image. The contour levels displayed are $-0.6,-0.42,-0.3,0.3$ $(=2 \sigma), 0.42,0.6,0.84, \ldots, 26.4$ mJy beam $^{-1}$, with a contour spacing of a factor of $\sqrt{2}$.

with the highest angular resolution in the sample, butbecause of the proximity of NGC 7090-also the spatial resolution is good $(1 \mathrm{kpc})$. The peak surface brightness of the averaged $z$ profile at $2.45 \mathrm{GHz}$ is only $4 \mathrm{mJy}^{\text {beam }^{-1}}$ and this map is accordingly the one with the smallest dynamic range (Fig. 17). Thus, the data used for calculating

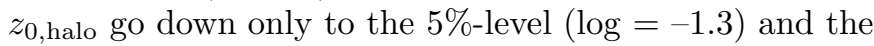
influence of noise is visible in the wings of the emission distribution.

\subsubsection{The $z$ structure of the disk emission}

The values of the "disk scale height", $z_{0, \text { disk }}$, in Table 4 represent the apparent exponential scale height of the thin disk emission component. At the resolution of our data, and given the uncertainties in the values of the inclination angles, $i$, it is not clear what causes the apparent thickness of the thin disk component in each galaxy. The total width could be due to either an inclination of $i<90^{\circ}$ or intrinsic disk thickness, or a superposition of both. The $z_{0 \text {,disk }}$ values are thus not in all cases a direct measure of the intrinsic disk thickness.

A comparison of the best-fitting model of a thin disk with the observed cut perpendicular to the disk plane tells immediately whether there is any residual emission that cannot be explained by the single-component model 

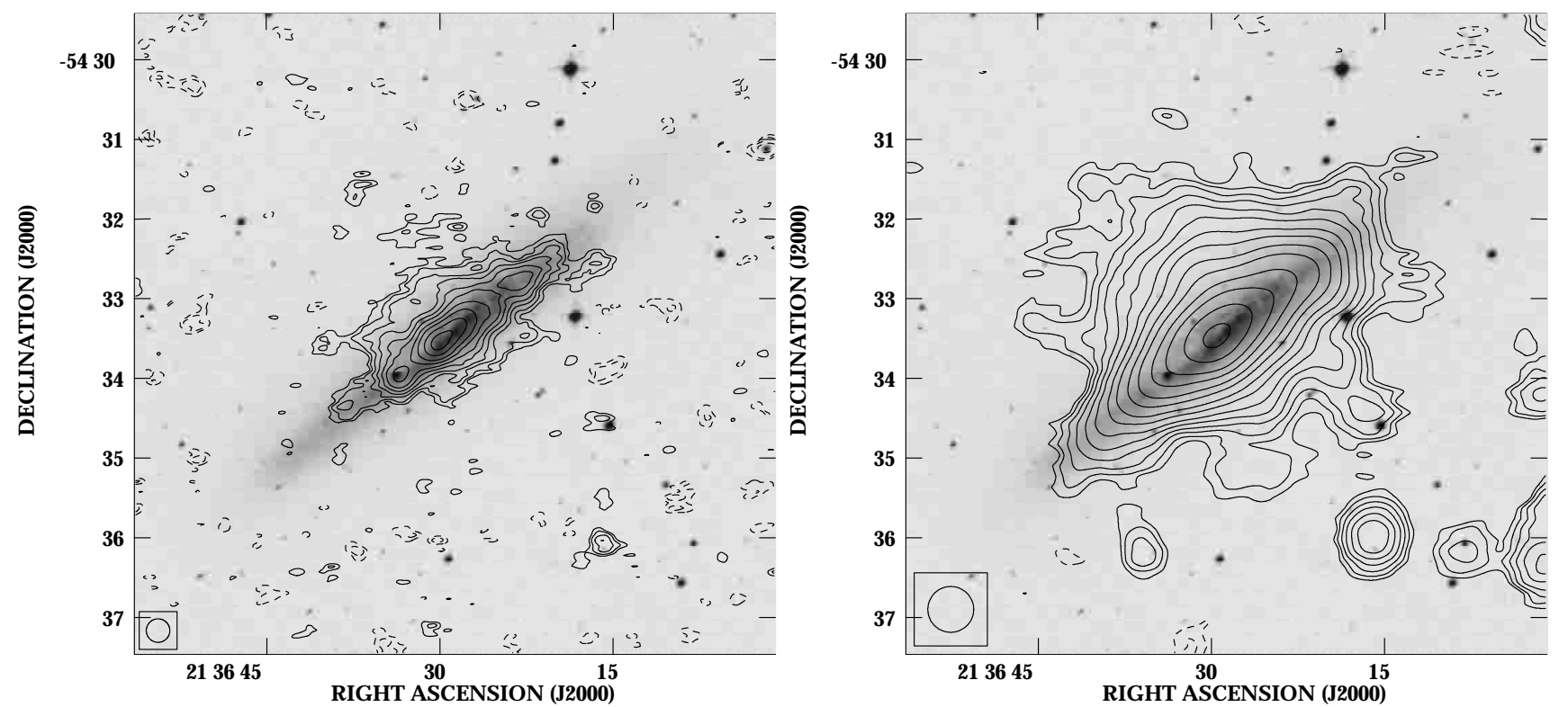

Fig. 13. ATCA radio continuum maps of NGC 7090. Left panel: $2.45 \mathrm{GHz}$ map; right panel: $1.43 \mathrm{GHz}$ map; both are overlaid on a DSS optical image. The contours display the $-4,-2.8,-2,2,2.8,4, \ldots \sigma$ confidence level (the $1-\sigma \mathrm{rms}$ is $0.075 / 0.05 \mathrm{mJy}$ beam $^{-1}$ at $2.45 / 1.43 \mathrm{GHz}$, respectively).
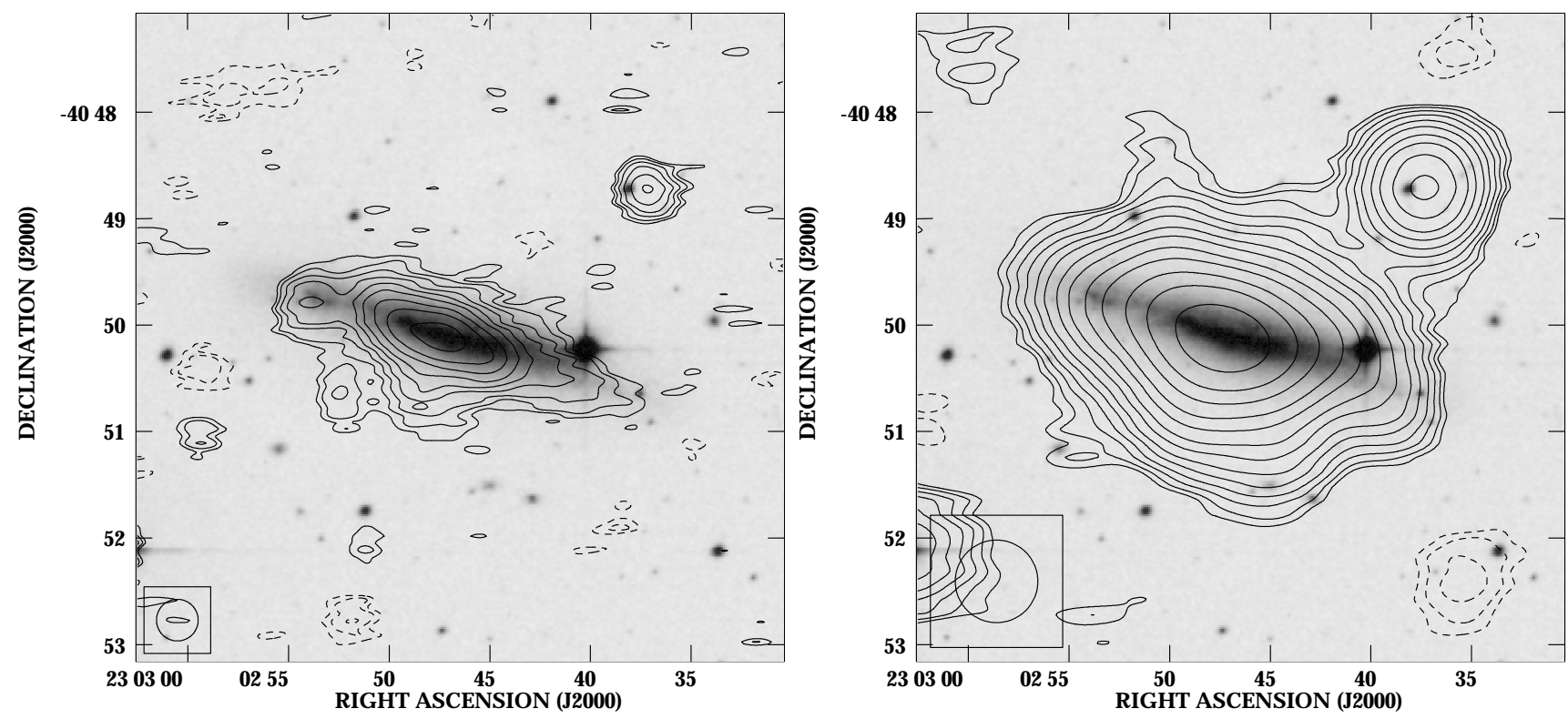

Fig. 14. ATCA radio continuum maps of NGC 7462. Left panel: $2.45 \mathrm{GHz}$ map; right panel: $1.43 \mathrm{GHz}$ map; both are overlaid on a DSS optical image. The contours display the $-4,-2.8,-2,2,2.8,4, \ldots \sigma$ confidence level (the $1-\sigma \mathrm{rms}$ is $0.085 / 0.05 \mathrm{mJy}$ beam $^{-1}$ at $2.45 / 1.43 \mathrm{GHz}$, respectively).

or not. Any emission at high projected $z$ offsets that is not well represented by the best-fitting single exponential (dotted line) must arise from outside the galaxy disk. In the presence of significant amounts of residual emission, especially in the form of broad wings in the emission distribution perpendicular to the disk plane, it is assumed that a radio halo exists. For all practical purposes, it is assumed that the galaxy disks are not warped in those parts from where the observed radio continuum emission arises. In a number of cases there is only marginal or no evidence for the existence of extraplanar synchrotron radio continuum emission. Some of our maps do not have sufficient resolution to draw firm conclusions yet. This affects in particular the most distant objects in the sample (NGC 5073 and NGC 7541), but also a few others.

\subsubsection{Search for radio halos and measurement of their properties}

In those cases where there is excess emission beyond the disk, the scale height of the second, wider exponential component is interpreted as the exponential scale height 


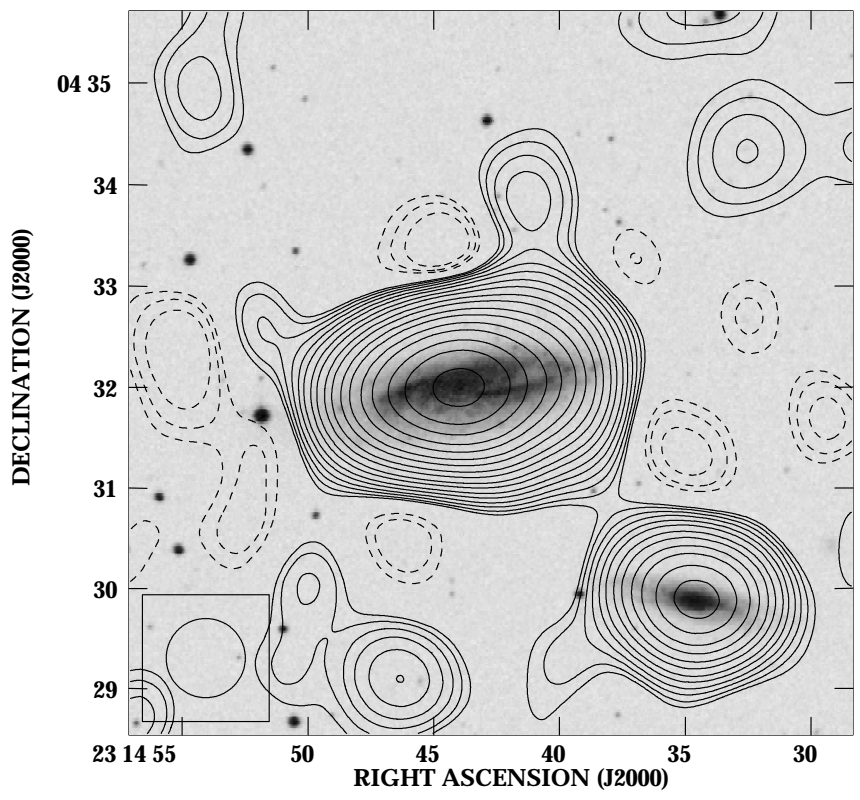

Fig. 15. VLA $1.43 \mathrm{GHz}$ radio continuum contour map of NGC 7541, superimposed on a Digital Sky Survey (DSS) image. The contour levels displayed are $-0.6,-0.42,-0.3,0.3$ $(=2 \sigma), 0.42,0.6,0.84, \ldots, 76.8 \mathrm{mJy}^{\text {beam }^{-1}}$, with a contour spacing of a factor of $\sqrt{2}$.

of the halo emission. It has been noted by different authors before that the surface brightness of radio halos declines exponentially away from the galaxy disks (see, for example, the high-resolution $z$ profile through NGC 891 by Dahlem et al. 1994). Therefore, the halo thickness can be quantified quite naturally by the exponential scale

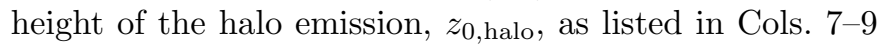
of Table 4. All other relevant parameters, including the relative amplitudes of the exponential components fitted and the radial ranges (Col. 3) over which the fits to the disk and halo emission components were performed, are tabulated there as well. Note that the relative amplitude of the two exponential components is no indication of the actual total flux percentages, because the $z$ profiles were performed only over a limited radial range, excluding the outer parts of the galaxy disks.

Data for the fits from which the exponential $z$ scale heights were determined (as listed in Table 4) were used only above the noise level of the images (Figs. 18-33). The threshold in all cases lies in the range from $1-5 \%$ of the peak surface brightness, depending on data quality and source brightness. In the following we will present a short description of the observed $z$ structure of all galaxies in the sample (except NGC 7090, which was already introduced above).

NGC 1055: There is no evidence in Fig. 18 for the presence of emission beyond the thin disk of NGC 1055. The beam-convolved exponential disk model is a good representation of the data. Therefore, the dotted line of the single-exponential component model and the dashed one of the total model overlap exactly, resulting in a dash-dotted line that in turn conincides in large parts with the solid line representing the data.

NGC 1406: Although relatively weak, the emission beyond the best-fitting disk model in the logarithmic plot in Fig. 19 is statistically significant and can be fitted only by adding a second exponential component. Higher angular resolution is required to confirm this preliminary result and to allow for a more reliable calculation of $z_{0 \text {,halo }}$.

NGC 1421: The $z$ profile of NGC 1421 (Fig. 20) does not give any indication of the presence of wings that cannot be fitted by a single-exponential model.

NGC 1511: Figures 21 and 22 display the $z$ profiles at $2.45 \mathrm{GHz}$ and $1.43 \mathrm{GHz}$, respectively. Both provide evidence for the existence of extraplanar radio continuum emission. The slopes of the halo emission are about linear in the logarithmic plots, indicating the expected exponential decay. Although the relative amplitude of the halo emission at $1.43 \mathrm{GHz}$ is only $4 \%$ of the peak, it is clearly detected (Table 4). The significantly lower amplitude of the halo component at $1.43 \mathrm{GHz}$ compared to $2.45 \mathrm{GHz}$ is most likely caused by beam smearing of disk emission.

NGC 2748: Figure 23 illustrates how precisely the emission profile of NGC 2748 can be approximated by a single beam-smeared exponential distribution.

NGC 2820: No proof of excess emission beyond the bestfitting disk model is visible in our data of NGC 2820 at the present resolution (Fig. 24).

NGC 3175: Although not very extended and at a low level, deviations from the best-fitting disk model were found on the south-west side of NGC 3175, which are modeled by an additional exponential component displayed in Fig. 25. In Sect. 4.2.1 radio data with a higher angular resolution are presented that clearly confirm the presence of a radio halo.

NGC 3437: There is no evidence for the existence of extraplanar emission from NGC 3437 from the $z$ profile in Fig. 26. Some low surface brightness excess emission is visible on the north side (positive offsets). However, the result is not clear enough to be considered a secure detection because of residual phase uncertainties in the data. Observations with higher resolution and better signal-tonoise ratio are warranted.

NGC 3717: NGC 3717 exhibits no deviations from the best-fitting disk model in Fig. 27, but note the large scale height of the emission distribution compared to those galaxies where disk and halo emission could be separated. 

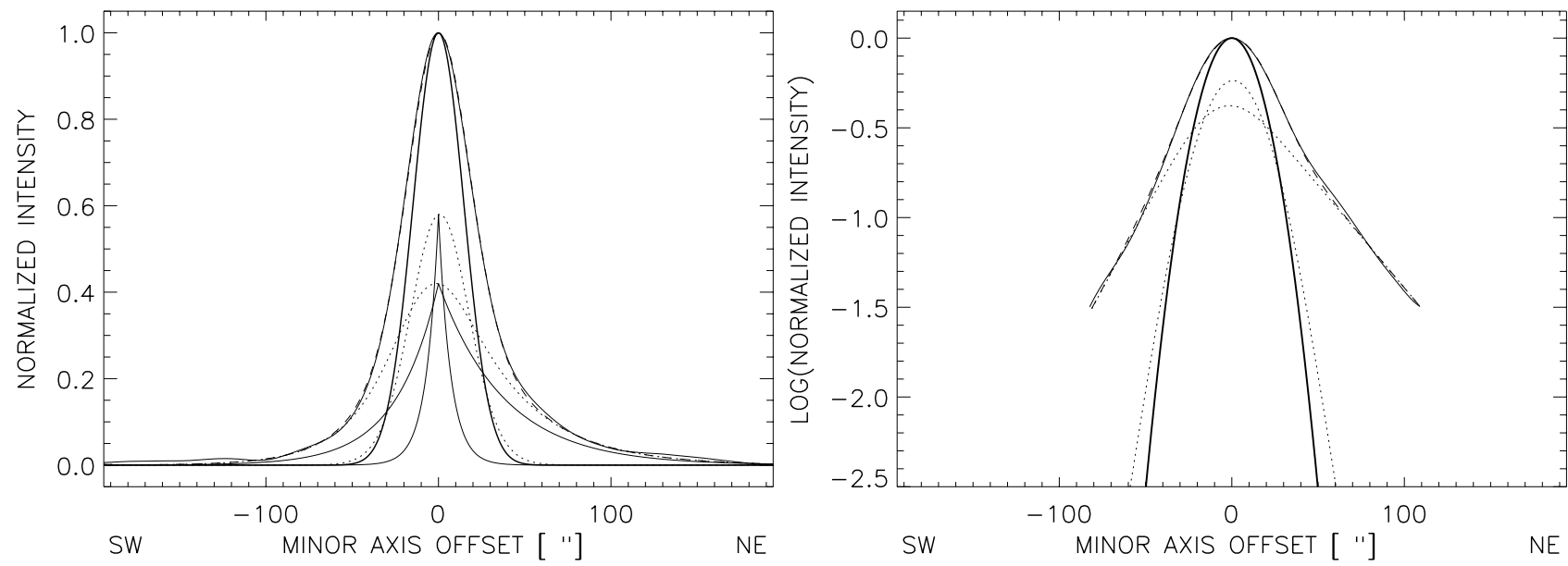

Fig. 16. Cut through the $1.43 \mathrm{GHz}$ emission of NGC 7090, perpendicular to the disk plane, on a linear scale (left) and logarithmic (right). In the linear graph the observed data (solid line), beam profile (bold line), exponential disk model and exponential halo model (solid lines, peaked distributions), the two beam-convolved exponential components (dotted lines) and the total model (sum of the two exponential components; dashed line) are presented. The logarithmic plot shows the same components, except the initial unconvolved exponentials.

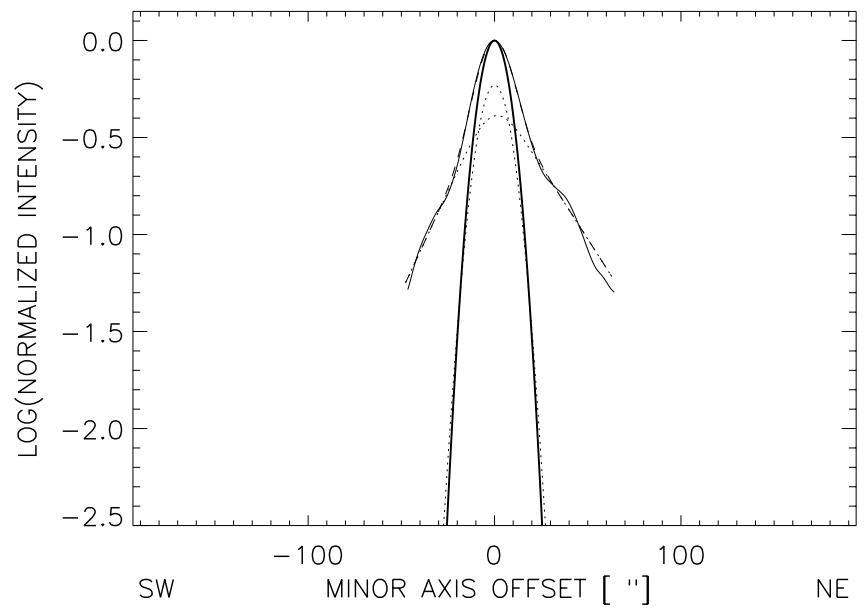

Fig. 17. Cut through the $2.45 \mathrm{GHz}$ emission of NGC 7090, perpendicular to the disk plane (same line types as the logarithmic plot in Fig. 16).

NGC 4527: In the case of NGC 4527 insufficient angular resolution is not the primary problem in the detection of halo emission (see Fig. 28). However, it has the widest apparent disk emission distribution of all objects in the sample, probably due to its relatively low inclination (see Col. 5 in Table 3). No significant emission beyond the disk model is detected. The slope of the measured data is partly even steeper than that of the model (especially on the north side), indicating that there is either a residual data problem or that the emission distribution falls off more steeply with increasing $z$-distance than an exponential.

NGC 4700: The most convincing new detection of a radio halo amongst the galaxies observed with the VLA D array has been made in NGC 4700, a starburst galaxy that had scarcely been studied prior to our observations. The

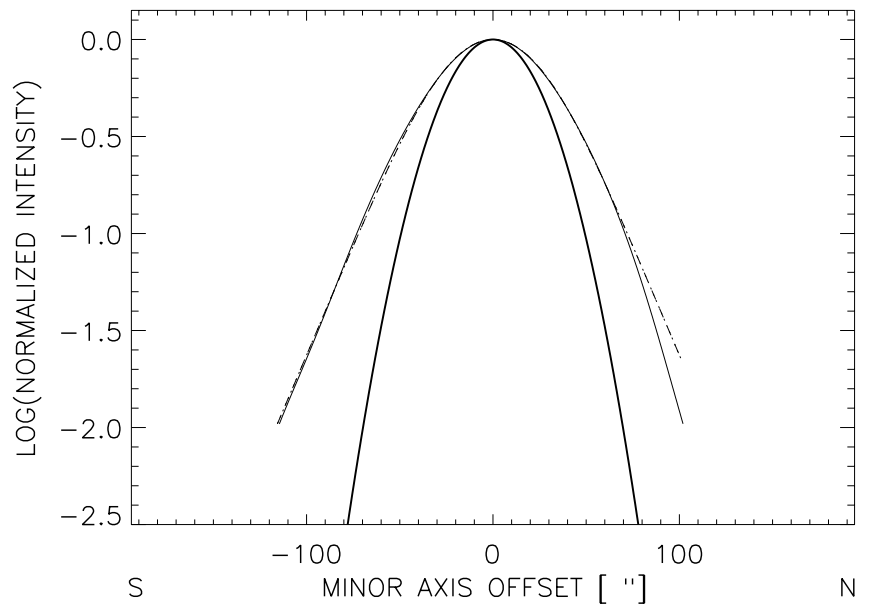

Fig. 18. Cut perpendicular to the disk plane of NGC 1055, perpendicular to the disk plane (same line types as in Fig. 17).

logarithmic $z$ profile in Fig. 29 exhibits wide linear wings on both sides of the galaxy disk, indicating the presence of an exponentially decaying $\mathrm{CR}$ halo that can be traced reliably out to the $1.25 \%$ level, up to 1.5 (10 kpc) away from the disk plane, with an unusually high scale height of $3.1 \mathrm{kpc}$. Deviations from the best fit to the disk emission start at a very high surface brightness. In fact, NGC 4700 is the only galaxy in the present sample in which the halo exponential has a higher amplitude than the disk component. The scale height of the disk component is not well-constrained because it is unresolved.

NGC 5073: This is the most distant object in our sample. No halo emission could be detected from this galaxy and the $z$ profile of the disk is also almost unresolved by the beam (Fig. 30). The fact that the observed profile crosses the beam profile might indicate residual phase problems in the data or a slope that is steeper than exponential. 


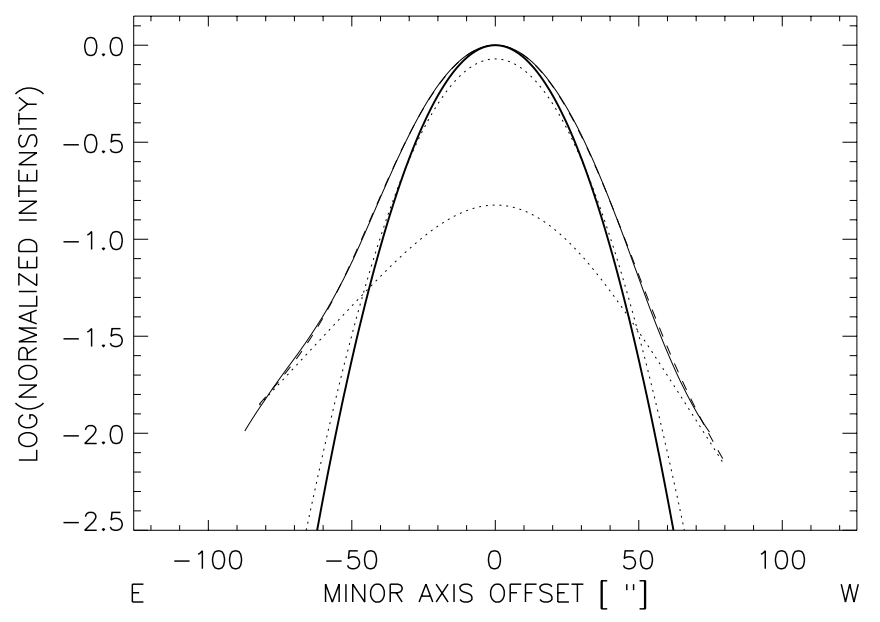

Fig. 19. Cut perpendicular to the disk plane of NGC 1406 (same line types as in Fig. 17).

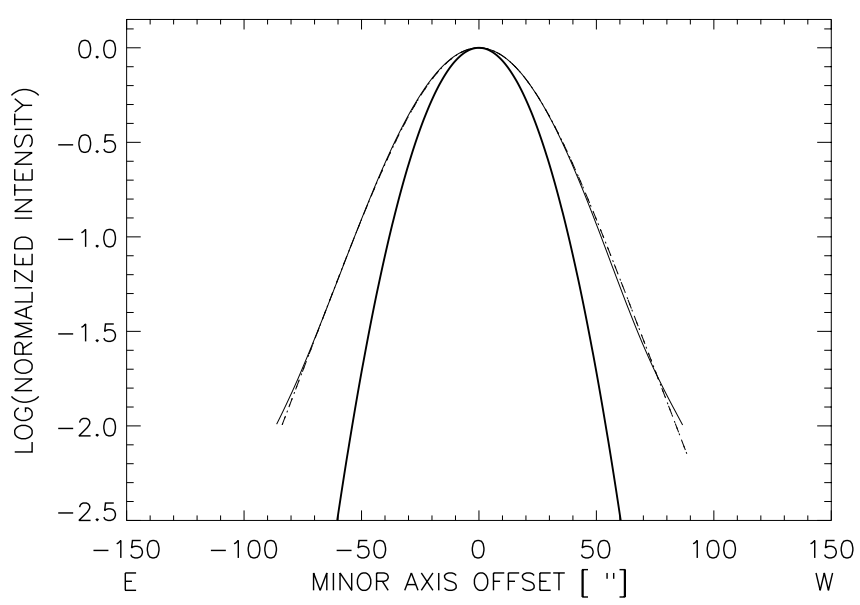

Fig. 20. Cut perpendicular to the disk plane of NGC 1421 (same line types as in Fig. 17).

NGC 7462: Clear signatures of extraplanar emission are detected in the $z$ profiles through NGC 7462 at both observing frequencies, $2.45 \mathrm{GHz}$ (Fig. 31) and $1.43 \mathrm{GHz}$ (Fig. 32). Also at both frequencies an asymmetry of the halo is apparent, the brighter emission being observed on the south side (towards negative offsets).

NGC 7541: This galaxy, at almost the same distance as NGC 5073, is also unresolved along its minor axis by our observations, as displayed in Fig. 33. No excess emission is observed beyond the exponential disk model.

\subsubsection{Summary of observations}

1. Halo emission was detected unambiguously in 6 galaxies out of 15 . The "raw" detection rate is therefore $40 \%$;

2 . the values of the exponential scale heights, $z_{0 \text {,halo, of }}$ the detected radio halos range from 1.4 to $3.1 \mathrm{kpc}$;

3. the data of all other galaxies can be approximated by a single-component exponential $z$ distribution model.

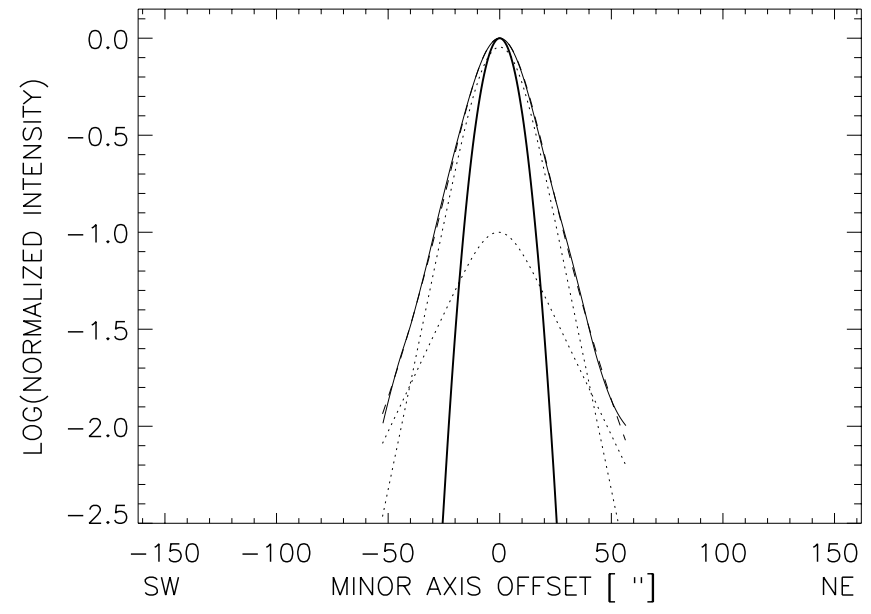

Fig. 21. Cut through the $2.45 \mathrm{GHz}$ emission of NGC 1511, perpendicular to the disk plane (same line types as in Fig. 17).

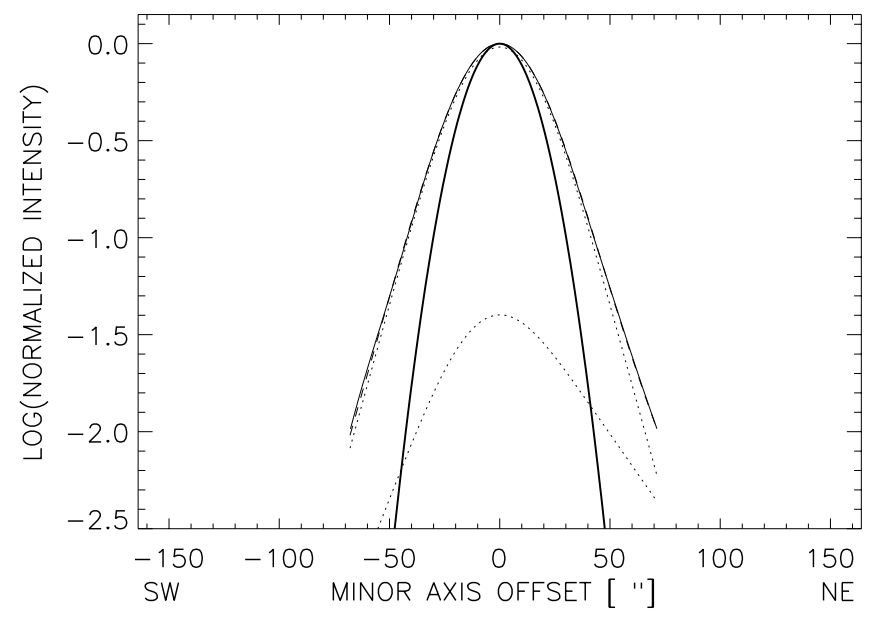

Fig. 22. Cut through the $1.43 \mathrm{GHz}$ emission of NGC 1511, perpendicular to the disk plane (same line types as in Fig. 17).

Table 5. Radio spectral indices from ATCA data.

\begin{tabular}{ccc}
\hline Galaxy & $\begin{array}{c}2.45 \mathrm{GHz} \\
\text { flux }(\mathrm{mJy})\end{array}$ & $\begin{array}{c}\bar{\alpha}_{1.43}^{2.45} \\
\left(S \propto \nu^{\bar{\alpha}}\right)\end{array}$ \\
\hline NGC 1511 & $101 \pm 5$ & $-0.81 \pm 0.18$ \\
NGC 7090 & $24 \pm 3$ & $-0.86 \pm 0.43$ \\
NGC 7462 & $16 \pm 2$ & $-0.91 \pm 0.45$ \\
\hline
\end{tabular}

Our $1.43 \mathrm{GHz}$ (and $2.45 \mathrm{GHz}$ ) radio continuum observations are therefore a suitable tool for searches of gaseous halos around spirals.

\subsection{Radio spectral indices}

From the VLA observations, and thus for 12 of the 15 galaxies, no spectral index information is available. However, the ATCA observations at two frequencies simultaneously do provide as a by-product spectral indices, $\alpha_{1.43}^{2.45}$. We have derived mean spectral indices, $\bar{\alpha}_{1.43}^{2.45}$, over the entire galaxies, as tabulated in Table 5. Mean values around $\alpha=-0.8$, as observed in the three galaxies 


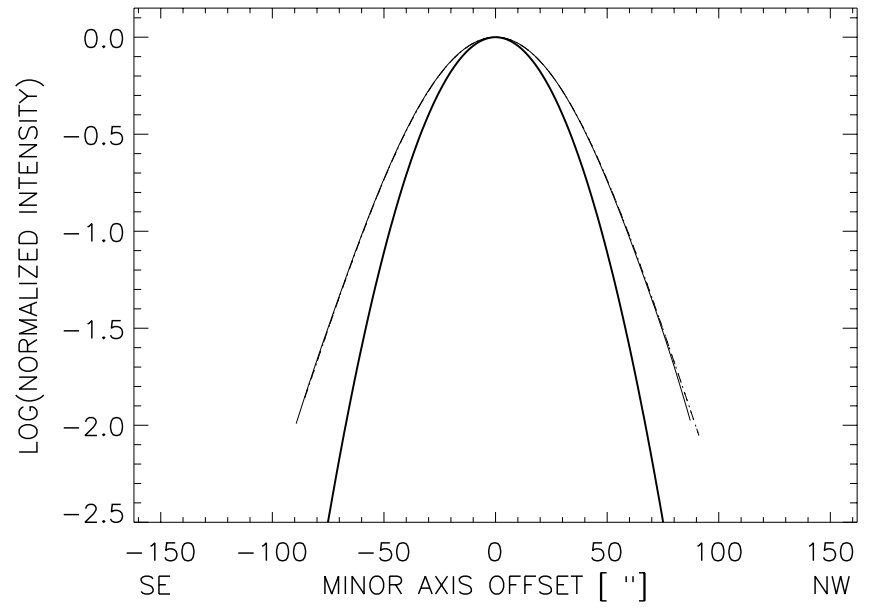

Fig. 23. Cut perpendicular to the disk plane of NGC 2748 (same line types as in Fig. 17).

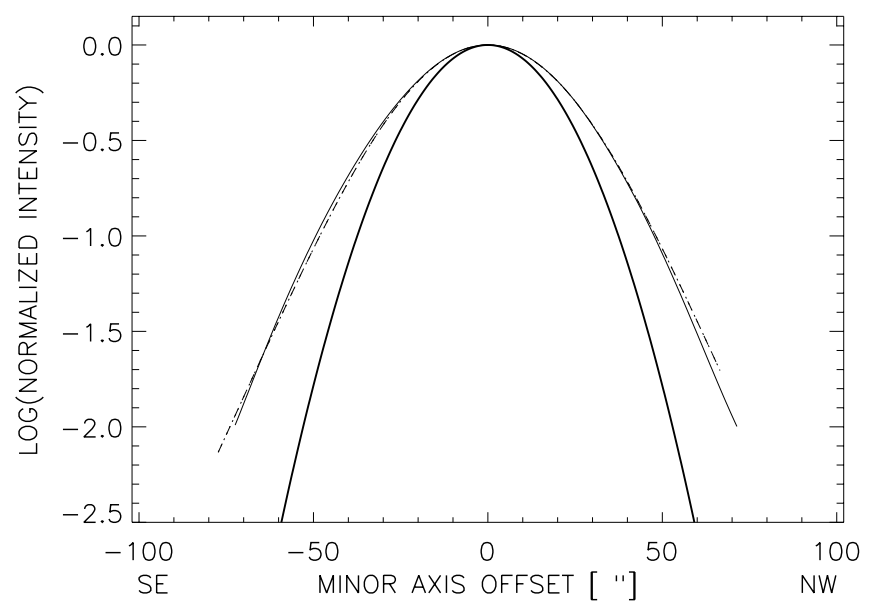

Fig. 24. Cut perpendicular to the disk plane of NGC 2820 (same line types as in Fig. 17).

NGC 1511, NGC 7090 and NGC 7462, are quite normal for late-type spiral galaxies (e.g., Lisenfeld \& Völk 2000 and references therein).

Images of the spectral index distribution within the three galaxies are displayed in Figs. 35-37. All three displays are presented in the same fashion, i.e., with the same grey scales and contours (see figure captions), making them directly comparable. The angular resolution of the spectral index maps is that of the $1.43 \mathrm{GHz}$ data. Spectral indices are displayed only in those areas where the signalto-noise ratio in both input images $(1.43$ and $2.45 \mathrm{GHz}$ ) is $\geq 5 \sigma$. The boundaries surrounding the spectral index distributions indicate that the maps were blanked outside this area.

The spectral index distribution in NGC 1511 (as displayed in Fig. 35) is quite homogeneous, with a few edge effects (especially at the southern and northern end of the distribution) that should not be taken at face value.

In NGC 7090 (Fig. 36) the flattest spectrum (i.e., most visibly influenced by thermal emission) is observed in the central part of the disk, with a slight steepening towards the outer disk and the halo regime.

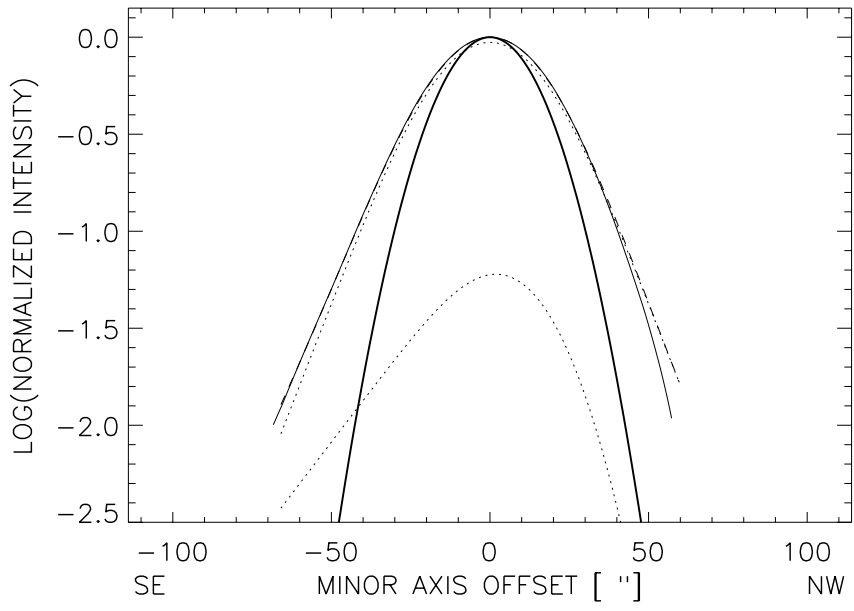

Fig. 25. Cut perpendicular to the disk plane of NGC 3175 (same line types as in Fig. 17).

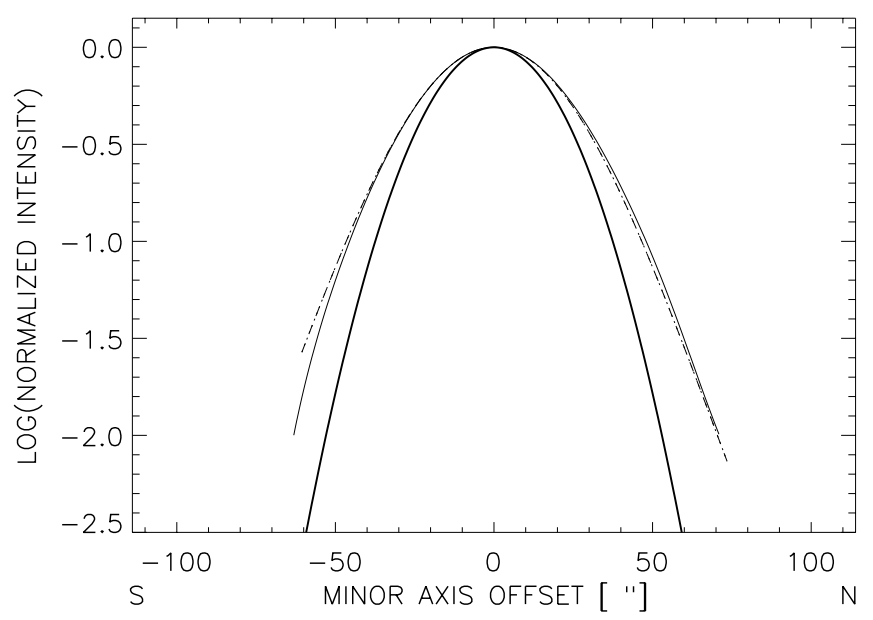

Fig. 26. Cut perpendicular to the disk plane of NGC 3437 (same line types as in Fig. 17).

NGC 7462 (Fig. 37) exhibits a spectral index steepening slightly away from the disk plane, with little substructure at the resolution of the current data.

A general trend is visible that the spectral indices steepen slightly away from the disk planes, indicating that energy losses of the CRs play an important role.

\subsection{Magnetic fields}

The maps in Figs. 1-15 show only total emission because, with the current sensitivity and angular resolution, no polarised radiation was detected (ATCA data) or calibration not performed (VLA observations). Thus, no information is available on magnetic field configurations in the observed galaxies. This implies that any possible effect that the magnetic field configuration might have on the observed CR distribution in the halos cannot be investigated here. 


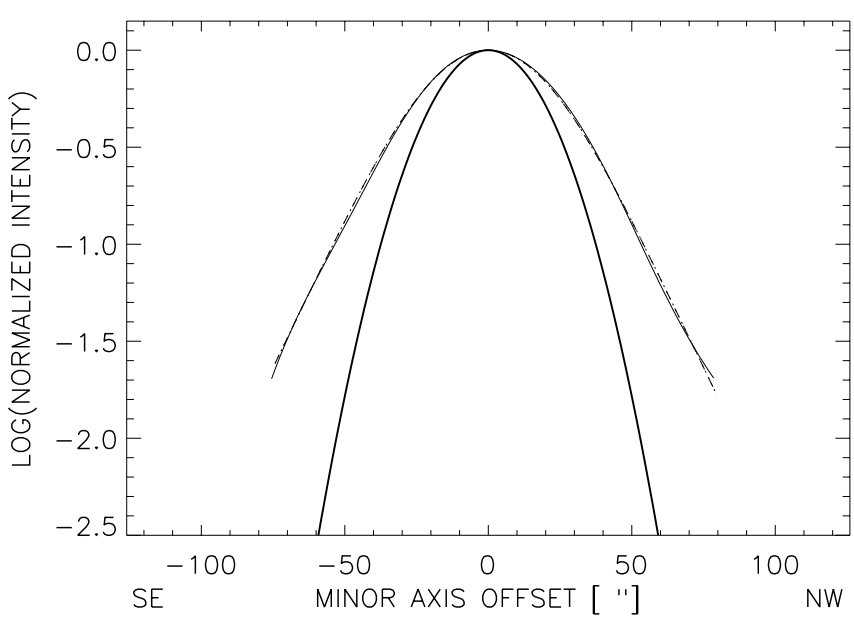

Fig. 27. Cut perpendicular to the disk plane of NGC 3717 (same line types as in Fig. 17).

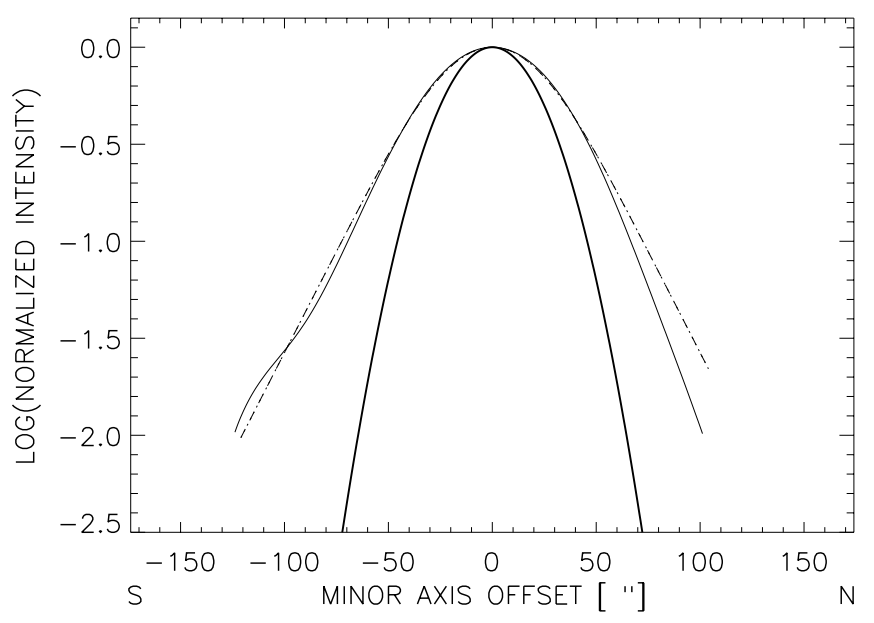

Fig. 28. Cut perpendicular to the disk plane of NGC 4527 (same line types as in Fig. 17).

\section{Discussion}

\subsection{Detections of radio halos from our data}

Four galaxies were excluded from the total sample of 15 objects for various reasons:

- In the case of NGC 2820 it was found that it has nearby interaction partners. Therefore, it is not clear whether energy input by active SF into its disk or a gravitational drag might dominate the energy balance of any observed disk-halo interactions;

- The inclination angle of NGC 4527 turned out to be too low to allow for the kind of studies intended here;

- Both NGC 5073 and NGC 7541 show signs of previously unlisted AGNs (see below), which we wanted to exclude from the beginning.

A "clean" sample of 11 galaxies remains. Of these the following 6 have positively detected radio halo emission: NGC 1406, NGC 1511, NGC 3175, NGC 4700, NGC 7090 and NGC 7462, see Table 6. For NGC 1055, NGC 1421,

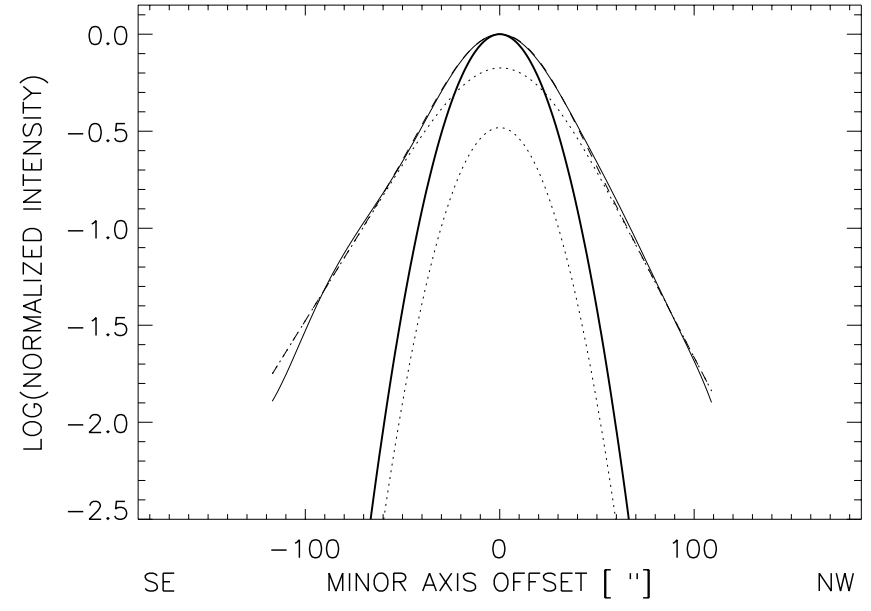

Fig. 29. Cut perpendicular to the disk plane of NGC 4700 (same line types as in Fig. 17).

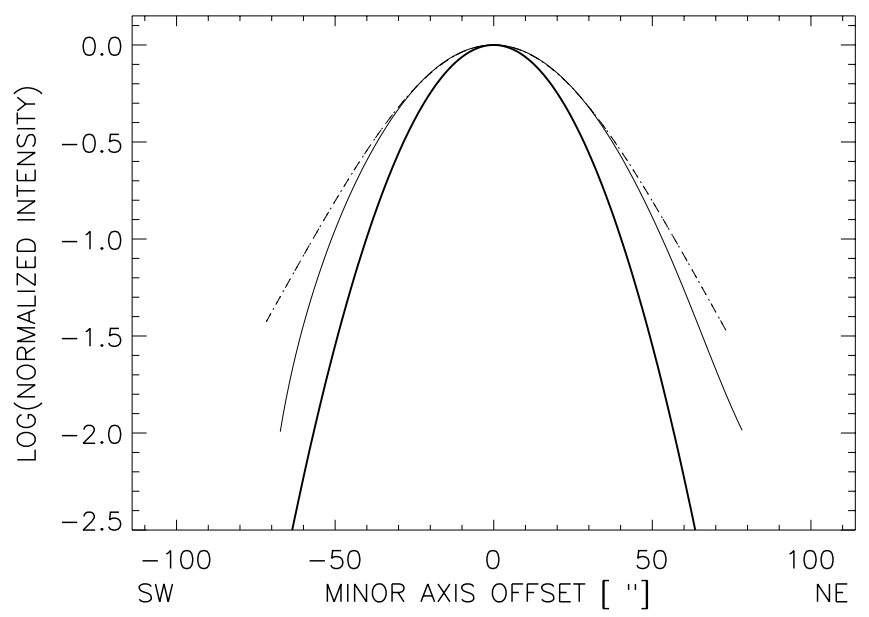

Fig. 30. Cut perpendicular to the disk plane of NGC 5073 (same line types as in Fig. 17).

Table 6. Detections of halo emission.

\begin{tabular}{ccc}
\hline Galaxy & Radio & H $\alpha$ \\
\hline NGC 1055 & no & - \\
NGC 1406 & yes & - \\
NGC 1421 & no & no \\
NGC 1511 & yes & yes \\
NGC 2748 & no & - \\
NGC 3175 & yes & no \\
NGC 3437 & no & - \\
NGC 3717 & no & - \\
NGC 4700 & yes & yes $^{a}$ \\
NGC 7090 & yes & yes $^{a}$ \\
NGC 7462 & yes & yes $^{a}$ \\
\hline
\end{tabular}

Note to Table 6: ${ }^{a}$ Rossa (2001).

NGC 2748, NGC 3437, NGC 3717, there is no or only marginal evidence. Thus, amongst the galaxies fulfilling the criteria for studies of dependences of their halo properties on their level of SF in the disks (i.e., those 


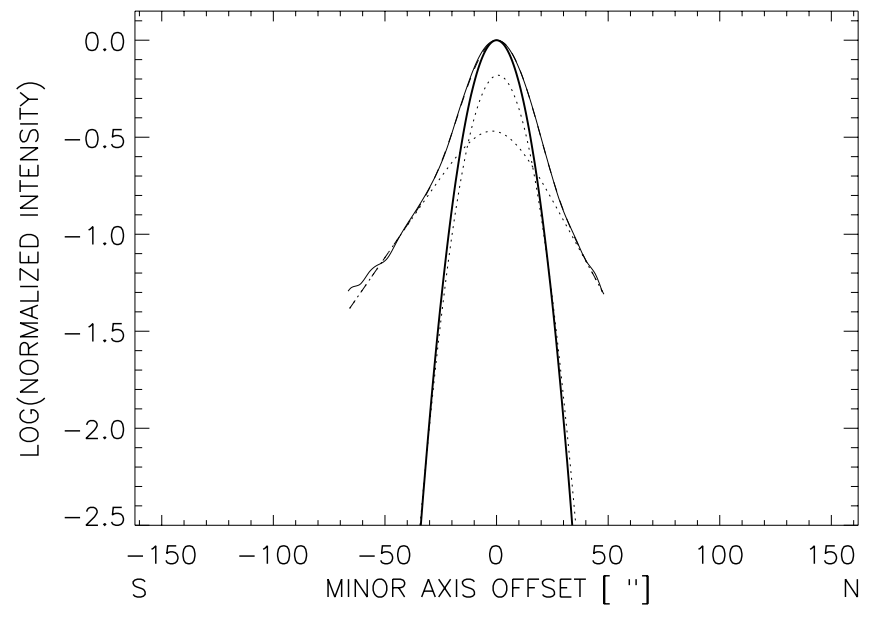

Fig. 31. Cut through the $2.45 \mathrm{GHz}$ emission of NGC 7462, perpendicular to the disk plane (same line types as in Fig. 17).

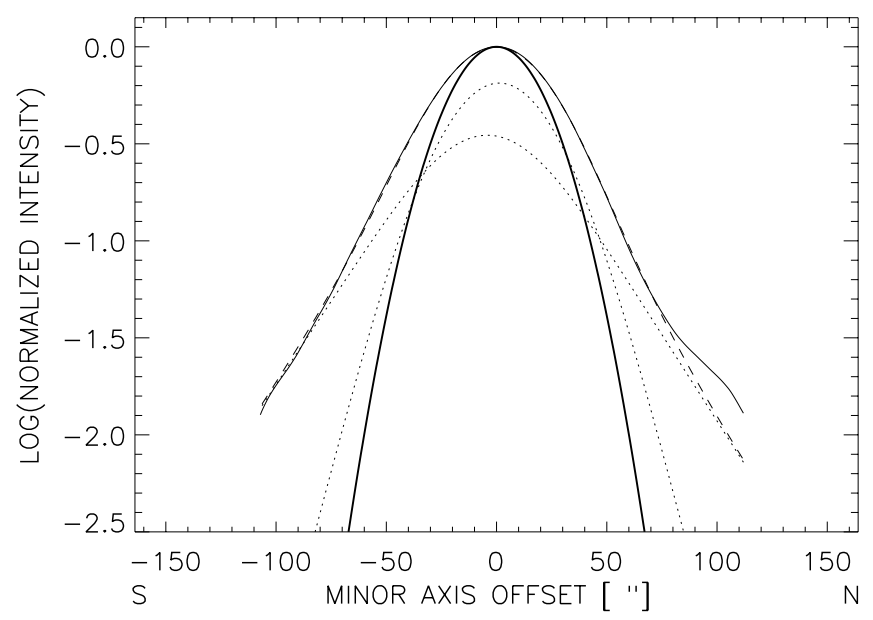

Fig. 32. Cut through the $1.43 \mathrm{GHz}$ emission of NGC 7462, perpendicular to the disk plane (same line types as in Fig. 17).

with $i \gtrsim 75^{\circ}$, no AGNs and no nearby interaction partners) the current detection rate is 6 out of 11 , i.e. $55 \%$.

The absence of clear evidence for the presence of halos in the remaining galaxies can, as indicated above, have different reasons:

- There is no halo because of too little energy input from the underlying disk;

- The inclination of the galaxy is too low. This cannot only lead to an apparently thick disk, but can also hamper the separation of residual high- $z$ emission from that widened disk;

- The angular resolution of our data is insufficient. The need for good angular resolution is demonstrated by a comparison of the two observations of NGC 3175 in Figs. 25 and 34 (more details are given in Sect. 4.2.1). From a marginal case in the former, twice the angular resolution leads to a clear detection with wellconstrained halo scale height measurements. In particular, the observations of NGC 3437 might be affected by this problem. Insufficient angular resolution and at

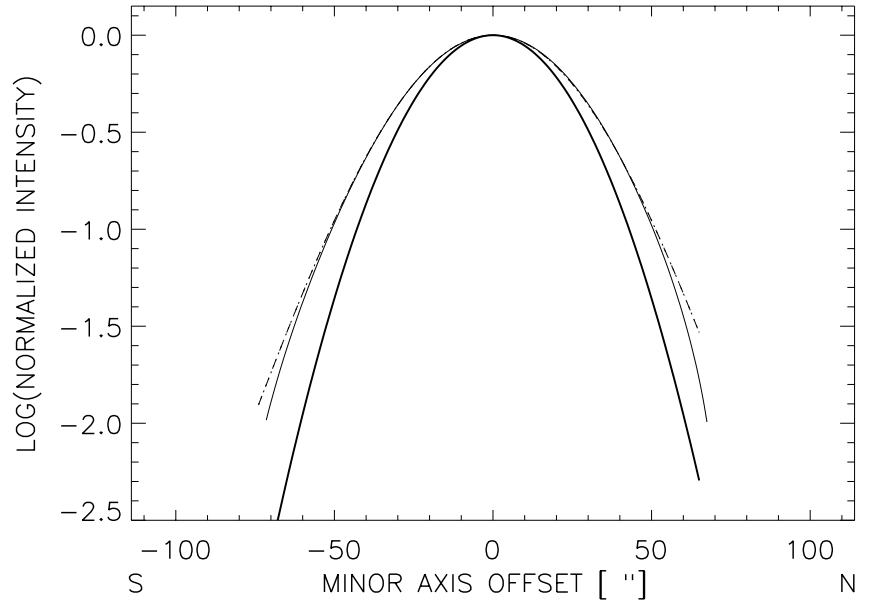

Fig. 33. Cut perpendicular to the disk plane of NGC 7541 (same line types as in Fig. 17).

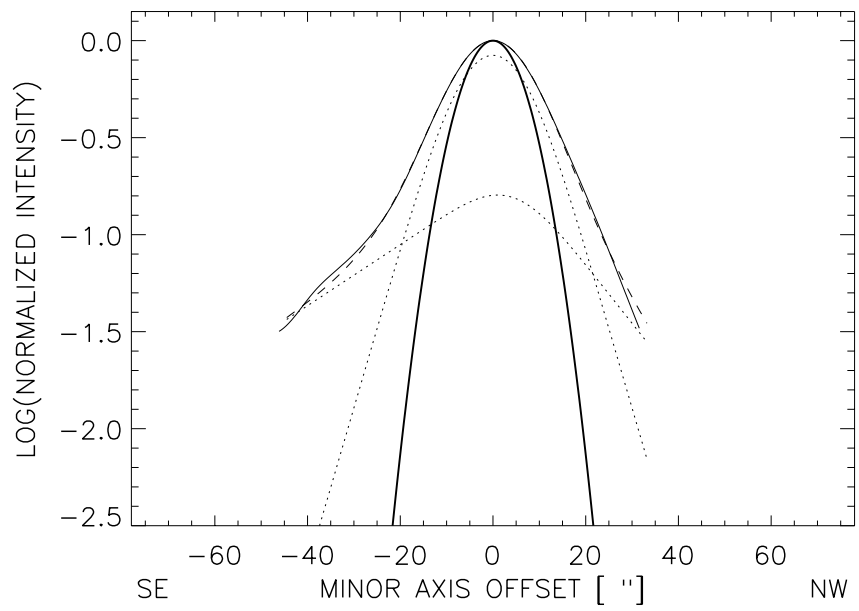

Fig. 34. Cut perpendicular to the disk plane of NGC 3175 (same line types as in Fig. 17). Based on VLA C array data by Condon et al. (1996).

the same time an inclination of $i<90^{\circ}$ can exacerbate the problem;

- The thin disk is not visible, because weak. Two of the galaxies in the present clean sample have $f_{60} / f_{100}$ ratios $>0.4$, but require only a single-exponential model. In principle, if one interprets this in terms of the absence of halo emission, this is a contradiction to our hypothesis. However, just these two galaxies require fits to their "disks" that are unusually wide compared to the thin disks in galaxies with spatially separable disk plus halo components (Table 4). This might suggest either a relatively low inclination angle, which can mimic the presence of a "thick" disk (but is unlikely because of the galaxies' optical appearance), or the presence of two unresolved components, of which the wide one dominates the overall emission distribution perpendicular to the disk. This is a reasonable explanation of our results, because the scale height of the $z$ distribution of NGC 3437 and NGC 3717, of 1.2 to $1.9 \mathrm{kpc}$, respectively, is more similar to those of the detected 


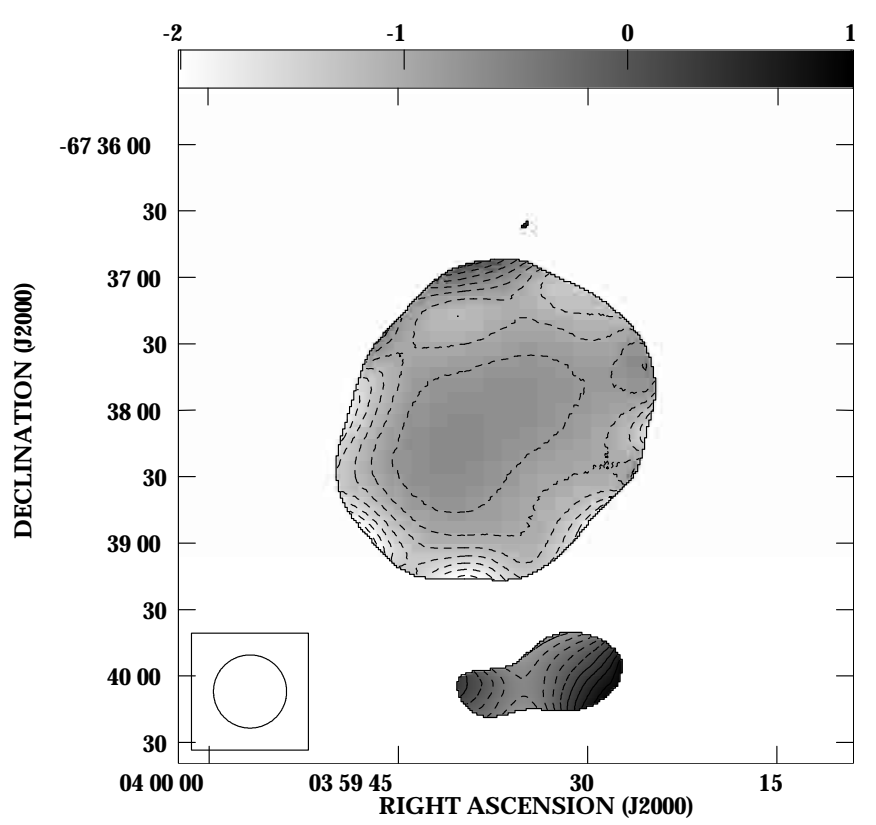

Fig. 35. $2.45 \mathrm{GHz}$ vs. $1.43 \mathrm{GHz}$ spectral index distribution within NGC1511. The grey-scale ranges from -2 to 1 ; the contours start at -2 , increasing by 0.2 each. The central oval contour represents a spectral index of -0.8 .

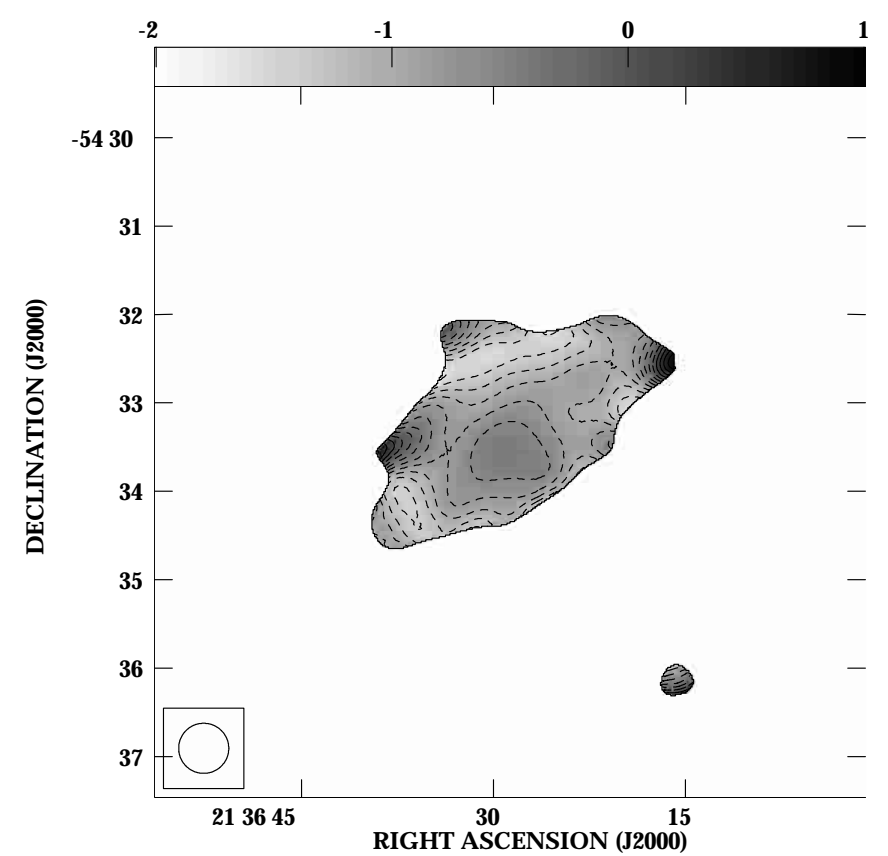

Fig. 36. $2.45 \mathrm{GHz}$ vs. $1.43 \mathrm{GHz}$ spectral index distribution within NGC 7090. The grey-scale ranges from -2 to 1 ; the contours start at -2 , increasing by 0.2 each. The central closed contour represents a spectral index of -0.6 .

halos than the scale heights of the detected thin disk components. Therefore, these galaxies might have radio halos that just require higher angular resolution to render possible the spatial separation of halo and thin disk emission.

Consequently, an FIR colour of $f_{60} / f_{100}>0.4$ is indeed a good search criterion for galaxies with a gaseous halo.

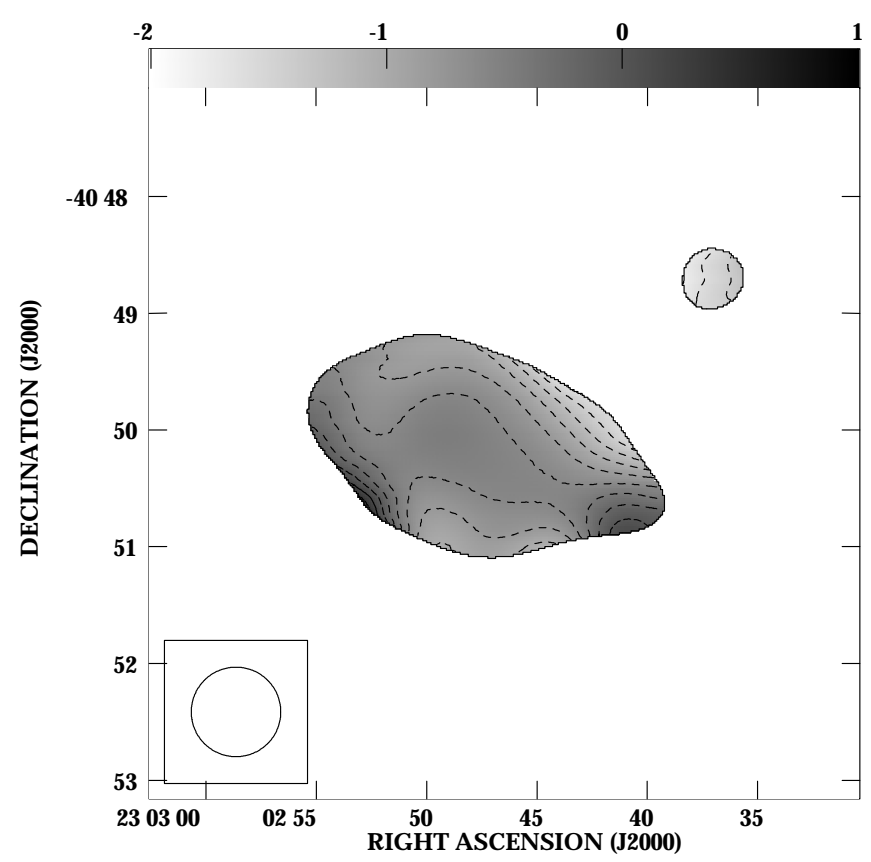

Fig. 37. $2.45 \mathrm{GHz}$ vs. $1.43 \mathrm{GHz}$ spectral index distribution within NGC 7462. The grey-scale ranges from -2 to 1 ; the contours start at -2 , increasing by 0.2 each. The central contours closest to the disk mid-plane represent a spectral index of -0.6 .

\subsection{Evidence for halo emission from different wavebands}

In addition to the synchrotron radio continuum from relativistic CR electrons there are other tracers of gaseous halos around spiral galaxies, of which the most important are $\mathrm{H} \alpha$ recombination radiation of warm ionised gas and soft X-ray emission from a hot thermal plasma (cf. Dahlem 1997).

\subsubsection{Previous radio observations}

In the following we briefly summarise those previously published radio continuum images that are relevant for searches of extraplanar emission in the galaxies presented here.

NGC 1421: Irwin et al. (1999) found some evidence for extraplanar emission in NGC 1421 from their VLA C array $1.4 \mathrm{GHz}$ radio continuum image (see their Fig. 4). The authors provided us with their data, which were combined with our D array visibilities to obtain a map that contains the full flux and has improved angular resolution (as displayed in Fig. 3).

NGC 2748: A $15^{\prime \prime}$ resolution $1.465 \mathrm{GHz}$ map of NGC 2748 by Hummel et al. (1985) might indicate the presence of radio continuum emission away from its disk plane (their Fig. 1c). However, these authors quote a lower total flux than measured by us. Due to missing short spacings (and 
thus missing extended flux) these data cannot be used for the present study.

NGC 2820: The VLA C array image by Hummel \& van der Hulst (1989) has a higher angular resolution than ours (15" [their Fig. 1] compared to $62^{\prime \prime} \times 41^{\prime \prime}$; Fig. 6 , above), but lower sensitivity. Based on a $z$ profile as used by us here (their Fig. 2), Hummel \& van der Hulst (1989) found that NGC 2820 has a thick radio disk or halo at $1.465 \mathrm{GHz}$. Since these authors did not report an exponential scale height for the halo emission, we rely here on our own measurement (note that this galaxy has been removed from the sample anyway). The fact that Hummel \& van der Hulst find evidence for the presence of a halo, while there is none in our data, demonstrates the need for sufficient angular resolution.

NGC 3175: A $1.49 \mathrm{GHz}$ radio continuum map of NGC 3175 by Condon et al. (1996) displays three maxima in the galaxy disk, one at the centre and one each about $45^{\prime \prime}$ on either side of it, thus coinciding with the most prominent H II regions in the $\mathrm{H} \alpha$ image by Ryder \& Dopita (1994). The contour map displayed in Condon et al. (1996) might also indicate halo emission above its central region. This VLA C array map has a higher angular resolution $\left(15^{\prime \prime}\right.$ compared to $\left.43^{\prime \prime} \times 33^{\prime \prime}\right)$ than ours, but lower sensitivity. It exhibits a low axial ratio of the radio continuum emission, suggesting that there might be a relatively small outflow from the nuclear region. Since it recovers the total flux (the same within the error margins as our measurement; see Table 3), Condon et al.'s image can be used for a study of the $z$ structure of the emission distribution.

Because of the very different integration times, it is not possible to combine Condon et al.'s data with ours. However, the image being available via NED, we have retrieved it and used it to produce a second $z$ profile. This higher-resolution $z$ profile is displayed separately in Fig. 34. The plot reassuringly reproduces the major features from Fig. 25, however in more detail. The distribution of excess emission beyond the thin disk is slightly asymmetric, being brighter on the south-east side (negative offset values), as already visible in our DnC array data. But now it is much clearer that this excess emission, in particular also that on the north-west side, is indeed significant. The results from the fitting process are collected in Table 4.

NGC 3437: Condon et al. (1990) presented a highresolution $1.49 \mathrm{GHz}$ image of NGC 3437. However, since it does not recover the total flux of the galaxy, it is not suitable for investigations of the extended emission distribution, including the $z$ structure perpendicular to the disk plane.
NGC 5073: We note here that a $1.49 \mathrm{GHz}$ radio image of NGC 5073 by Condon et al. (1990) exhibits a compact central source that is unresolved at a resolution of about $5^{\prime \prime}$. This is relevant in the context of the $\mathrm{H} \alpha$ data of this galaxy mentioned below. Since it does not recover the full extended flux of the galaxy, the radio map cannot be used for a study of the large-scale emission distribution along the minor axis.

NGC 7090: Two "spurs" of radio emission above the disk of NGC 7090 were detected by Harnett \& Reynolds (1985) at $843 \mathrm{MHz}$ (see their Plate 3). Our maps are more sensitive and have better angular resolution thus showing more extended halo emission. The brightest spur north-east of the central region, now embedded in extended emission, is visible in our $1.43 \mathrm{GHz}$ map (cf. Fig. 13) as kinks in the isophotal contours, which can be traced from close to the disk plane out to the detection limit of the halo.

NGC 7462: There is a tentative detection of extraplanar radio continuum emission from NGC 7462 in a $1.49 \mathrm{GHz}$ VLA map by Condon et al. (1987). However, the angular resolution (of $1^{\prime}$ ) and sensitivity of these snapshot observations were insufficient to make a firm statement. Our detection is much more conclusive, in particular because the result comes from observations at two independent frequencies.

NGC 7541: Condon et al. (1990) published a snapshot $1.49 \mathrm{GHz}$ map of NGC 7541, with a resolution of $15^{\prime \prime}$. It exhibits a dominant unresolved central source, which might hint at the presence of an active nucleus. Since this radio image does not recover all extended source flux, it is not used as part of the present investigation.

\subsection{2. $\mathrm{H} \alpha$ line imaging}

5 of the 15 galaxies studied here are also in the sample by Lehnert \& Heckman (1995): NGC 1511, NGC 2820, NGC 4527, NGC 5073 and NGC 7541. Three of these, namely NGC 1511, NGC 4527 and NGC 5073, were imaged in $\mathrm{H} \alpha$ line emission. In addition, $\mathrm{H} \alpha$ images of a few more individual galaxies can be found in the literature.

NGC 1421: An unpublished $\mathrm{H} \alpha$ image by Dettmar (priv. comm.) exhibits numerous HII regions in the disk of NGC 1421, indicating a two-arm spiral structure and an inclination of $i<90^{\circ}$. No extraplanar $\mathrm{H} \alpha$ line emission was detected. However, this might be due to a lack of sensitivity.

NGC 1511: The H $\alpha$ image of NGC 1511 by Lehnert \& Heckman (1995) shows a disturbed structure, with HII regions that are located outside the thin disk plane. There are also indications of diffuse $\mathrm{H} \alpha$, which apparently does 
not arise from the central disk plane, but there is no direct evidence for halo emission. The disturbance of its disk might arise from interactions with one of its partners, NGC 1511A or $B$, or just be its intrinsic structure. With an absolute $R$ magnitude of -18.6 (Lehnert \& Heckman 1995) NGC 1511 is a low optical luminosity, low-mass galaxy, in which disturbances are known to be quite normal (compare, e.g., with NGC 1569, NGC 4449 or NGC 2188).

NGC 3175: An H $\alpha$ image by Ryder \& Dopita (1994) shows bright $\mathrm{H}$ II regions at the position of the nucleus and what appears to be a starburst ring with a radius of $3.5 \mathrm{kpc}$ around it. The HII regions at the tangential points of this ring are apparently the brightest. These might also be "thick", i.e. extended in the direction perpendicular to the disk plane. However, no extended diffuse $\mathrm{H} \alpha$ emission is visible, especially away from the disk plane. Note that NGC 3175 does not exhibit any signs of ongoing star formation outside the starburst ring. Its outer disk has a very red colour (Ryder \& Dopita 1994) and is H I deficient (Dahlem et al. 2001).

NGC 4527: The H $\alpha$ image of NGC 4527 by Lehnert \& Heckman (1995) shows no obvious signs of extraplanar emission. Instead, it indicates that the disk is not inclined exactly edge-on. From its optical axial ratio of 3 Tully (1988) derived an inclination angle of about $i=68^{\circ}$, which has been taken into account in our representation of the radio emission from the disk in Fig. 28 and which is the reason for removing the object from the present sample.

NGC 4700: NGC 4700 was observed by us (Rossa 2001) and the resulting image will be presented separately. Clear evidence for extraplanar $\mathrm{H} \alpha$ emission is visible in our frame; therefore the corresponding entry was made in Table 6 .

NGC 5073: In the $\mathrm{H} \alpha$ image of NGC 5073 by Lehnert \& Heckman (1995) one can see little diffuse emission above the nuclear area and a strong point source in the centre. Together with the unresolved nuclear radio continuum source, this might hint at the presence of a previously unclassified active nucleus, which could explain why a high $f_{60} / f_{100}$ flux ratio is observed and the radio emission is unresolved by our data, while there is no indication of a halo.

NGC 7090 and NGC 7462: Both galaxies were observed and extraplanar $\mathrm{H} \alpha$ emission clearly detected (Rossa 2001). These results will be presented at a later time, together with those on NGC 4700.
Resume: The small number of $\mathrm{H} \alpha$ detections is probably in most cases due to sensitivity problems, because the images available in the literature were not taken for dedicated searches of low surface brightness extraplanar emission. Long integration times are required for attempts to detect such emission.

\subsubsection{Soft X-ray imagery}

Data existing in archives. ROSAT archival data exist for NGC 1406, NGC 2820, NGC 4527; however, they do not contribute anything new to the current investigation. NGC 1406 is detected, but unresolved; a by-eye inspection of the data reveals no detected emission from either NGC 2820 or NGC 4527.

Einstein observations of NGC 1421 show weak emission from its disk, but also from a "tail" to the south of the optically visible disk and also west of the nuclear area, thus perpendicular to the disk plane, where the extreme colour stretch used by Fabbiano et al. (1992) for the underlying optical image might suggest the presence of a stellar tidal arm.

Future observations. Of the present sample, Chandra observations of NGC 1055 have been approved. We will obtain XMM-Newton Guaranteed Time observations of NGC 1511 and NGC 5073, which will be presented elsewhere in due course.

\subsubsection{Summary of observational evidence}

Considering all observations listed above of tracers of gaseous halos, 6 out of the 11 galaxies presented here show signs of extraplanar emission in at least one waveband. Different components of the halo ISM in the target galaxies were detected in four cases, as previously done in more nearby systems (see e.g. Dahlem 1997). A list of the detection/non-detection of extraplanar radio and $\mathrm{H} \alpha$ emission is provided in Table 6 .

Thus, it appears that the existence of gaseous halos around late-type spiral galaxies is indeed not as uncommon as it appeared in the past. Rather, the search criteria are now optimised. Also the high detection rate by Irwin et al. (1999) of $>90 \%$ fits into this scenario.

FIR brightness and in particular "warm" FIR colours, i.e. high $f_{60} / f_{100}$ flux ratios, have turned out to be a very effective search criterion (Heckman et al. 1990; Lehnert \& Heckman 1995). This is also born out by the fact that all FIR-warm nearby edge-on starburst galaxies studied by us previously in the soft X-ray regime (D98) exhibit diffuse extraplanar X-ray emission. A somewhat lower detection rate in the present sample can be expected, because we include here objects at larger distances and thus with lower flux densities and surface brightnesses than before. With values of in some cases 5-9 kpc (Table 4) also the spatial resolution of our data is not always sufficient yet. 


\subsection{On the origin of gaseous halos}

\subsubsection{Making sure that star formation dominates the energy input into the ISM}

As outlined by Dahlem (1997), several processes can contribute to the creation of gaseous halos around late-type spirals. In order to make sure that only star-formation related energy input is taken into account, galaxies with AGNs and closely interacting systems have been removed from the original sample above. Many starburst galaxies are interacting, or reversely, there is an overabundance of starbursts in interacting galaxies (cf. Hummel et al. 1990; Lutz 1992). In the case of distant encounters one can hope that the interaction has only caused minor disturbances of the gas in the galaxies, leading to the accretion of gas near their inner Lindblad resonance (Combes 1987) or turnover point of galactic rotation (Lesch et al. 1990) and subsequently to the onset of the observed starbursts, while the starbursts themselves dominate the energy balance at the time of the observation. This leaves galaxies that have high $f_{60} / f_{100}$ flux ratios because of SF-related heating of their dust, with approximately unperturbed gravitational potentials. SF-related heating sources are photo-ionisation, shocks and/or turbulent mixing layers, but in principle all energy comes from processes relating to the evolution of high-mass stars (winds and type II supernovae; Leitherer \& Heckman 1995; Leitherer et al. 1999). The above points out that careful selection of candidates and interpretation of the results is crucial; too many free parameters would leave doubts about the source of the energy driving the gaseous halos. Based on the above selection, we are confident that halo emission detected in the remaining 11 galaxies of our sample arises from energy input into the ISM from high-mass stars and type II SNe.

\subsubsection{Measuring techniques}

Having ensured that SF dominates the energy input into the disk ISM, the next step in an investigation of a potential connection between halo properties and SF activity in the underlying disk is to quantify the level of energy input and the halo properties.

In earlier studies of halo properties (e.g. Hummel et al. 1991b; Dahlem et al. 1994; Dahlem et al. 1997) we have used $z$ profiles averaged over a broad radial range to quantify the properties of radio halos. This has the advantage of increasing the signal-to-noise ratio compared to a single-pixel profile. At the same time, data are only averaged over regions where emission in the halo has clearly been detected, adapting the measurements to the individual target's geometry.

Ellipsoidal fitting to radio images, as performed by Irwin et al. (1999) makes sense under the assumption that radio halos are engulfing their host galaxies. However, this is not observed in images with sufficient angular resolution. Images that might suggest such behaviour (like, for example, our $1.43 \mathrm{GHz}$ map of NGC 1511 in Fig. 4) often suffer from insufficient angular resolution. Instead, with good resolution, a behaviour as described by us earlier (DLG95) is observed in most galaxies: radio halos exist only above the brightest emission regions of the underlying galaxy disks, i.e. the active $\mathrm{SF}$ regions. The radial extent of the halos is similar to the radius out to which SF is observed, $r_{\mathrm{SF}}$, and thus smaller than that of the entire disks. This is evident in our new $1.43 \mathrm{GHz}$ and $2.45 \mathrm{GHz}$ images of NGC 7090 (Fig. 13). Supporting evidence for this result comes from recent $\mathrm{H} \alpha$ observations by Rossa \& Dettmar (2000). Ellipses do not fit the geometry of the halo plus disk emission properly. We find that the most reliable technique to detect and quantify extraplanar emission is by producing averaged $z$ profiles.

\subsection{Dependence of halo properties on the level of disk activity?}

\subsubsection{Quantifying the energy input into the disk ISM}

Based on radio data of the nearby edge-on galaxies NGC 891 and NGC 4631, and - with less detailed information - on a few other galaxies, we started investigating whether such a dependence exists (DLG95). As a measure of the energy input into the disk ISM per unit time, $\dot{E}$, we used the nonthermal radio continuum emission produced by type II SNe. In order to obtain a good measure of the energy density in a given volume within the galaxy disk, the area over which energy input by active $\mathrm{SF}$ takes place, $A_{\mathrm{SF}}$, was quantified by us. Determining from radial profiles of the radio continuum emission distribution in the disk a cut-off radius of SF processes, $r_{\mathrm{SF}}$, we calculated, adopting circular symmetry, $A_{\mathrm{SF}}$, as the circular area within the disk of the galaxies over which SF-related energy production occurs, following the simple relation $A_{\mathrm{SF}}=\pi r_{\mathrm{SF}}^{2} \cdot r_{\mathrm{SF}}$ can be determined equally well from the radial distribution of radio continuum emission in the disk. Data of in total 9 galaxies indicated a trend that those galaxies with the highest energy injection rates, $\dot{E} / A_{\mathrm{SF}}$, per unit time and unit surface area, have the most prominent radio halos (DLG95). Below a certain energy input level no outflows are enabled and galaxies thus do not exhibit extraplanar radio emission. Similar results were reached by Rand (1996) and Meurer et al. $(1995,1997)$, based on data from other wavebands. A possible link between high energy input rates into the disk ISM and the existence of soft X-ray halo emission is discussed by Ehle et al. (1998) and Dahlem et al. (1998).

The remaining 11 galaxies in the present sample can also be studied for such a dependence. The relevant properties are listed in Table 7 . We use as a measure of $r_{\mathrm{SF}}$ the beam-deconvolved radial extent of the $1.43 \mathrm{GHz}$ radio continuum emission from our current observations. We limit our studies here to the global energy input as a measure of which we will use the total FIR luminosity $L_{\mathrm{FIR}}$, as done by Rossa \& Dettmar (2000). Based on the total FIR flux, FIR, following the relation FIR $=1.26\left(2.58 f_{60}+f_{100}\right)$, we calculate $L_{\mathrm{FIR}}=4 \pi D^{2}$ FIR. $L_{\mathrm{FIR}} / A_{\mathrm{SF}}$ is the FIR 
luminosity normalised per unit surface area of the starforming part of the disk, representing the energy input rate $\left(\dot{E} / A_{\mathrm{SF}}\right)$, as defined similarly by us based on radio surface brightnesses (DLG95). This quantity is used as a measure of the global rate of energy injection into the ISM via SF-related processes (stellar winds and type II

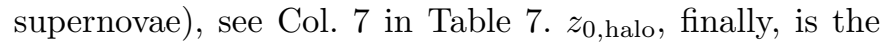
exponential scale height of the radio halos from Table 4 .

Rossa \& Dettmar (2000) have made further progress in another direction, plotting the $f_{60} / f_{100}$ flux ratio vs. the total FIR luminosity (as a measure of $\dot{E}$ ) normalised by the disk surface area, $L_{\mathrm{FIR}} / D_{25}^{2}$ (in particular, their Fig. 13), where they find a clear distinction between quiescent and starburst galaxies. All galaxies classified as starbursts by their location in the $f_{60} / f_{100}$ vs. $L_{\text {FIR }} / D_{25}^{2}$ plot (their Fig. 13) have detections of $\mathrm{H} \alpha$ emission from extraplanar diffuse ionised gas (eDIG). Compared to DLG95, the FIR luminosity, $L_{\mathrm{FIR}}$, substitutes the radio luminosity to represent the level of energy injection, $\dot{E}$. As a first approximation, Rossa \& Dettmar use in their determination of the surface area of the energy input the 25 th magnitude isophotal diameter, $D_{25}$. For the galaxies with wide-spread SF over their disks as used in their sample, this approximation is acceptable. However, in other cases-especially the classic starburst galaxies with circumnuclear activity-the use of $D_{25}$ can lead to serious errors: systems like, e.g., NGC 1808 actively form stars over a much smaller area than their disks out to $D_{25}$ (e.g., Dahlem et al. 1990). In the present sample NGC 3175 is such a case. While its $D_{25}$ is $5^{\prime}$, the diameter of the star-forming part of its disk can be determined (from $\mathrm{H} \alpha$ and radio continuum images; see Condon et al. 1996; Ryder \& Dopita 1994) very reliably to be only $90^{\prime \prime}(7 \mathrm{kpc})$. Thus, using $D_{25}$ would lead to an over-estimate of the surface area over which its energy input is distributed, by a factor of 11.1 and thus underestimate the energy density in the disk by the same factor. In these cases one must determine the area over which SF takes place more accurately, by using $r_{\mathrm{SF}}$ and $A_{\mathrm{SF}}$ instead.

\subsubsection{The ubiquity of gaseous halos in FIR-warm spirals}

Figure 38 corresponds with Figs. 11 and 12 by Rossa \& Dettmar (2000). It displays the $f_{60} / f_{100}$ FIR flux ratio (thus a measure of the mean dust temperature) vs. the total FIR luminosity, normalised with the area in which active SF is taking place. In this logarithmic plot all galaxies occupy the upper right-hand quadrant where, according to Rossa \& Dettmar (2000) the starburst galaxies fall. Based on our selection criteria the bottom left-hand corner of the plot is empty; there are no quiescent galaxies in the sample. Amongst the galaxies plotted in Fig. 38 a trend is visible that the galaxies with the highest $\dot{E} / A_{\mathrm{SF}}$ values have the highest $f_{60} / f_{100}$ flux ratios and thus mean dust temperatures.

The new result of our investigation is that in 6 out of 11 objects radio halo emission has been detected (open

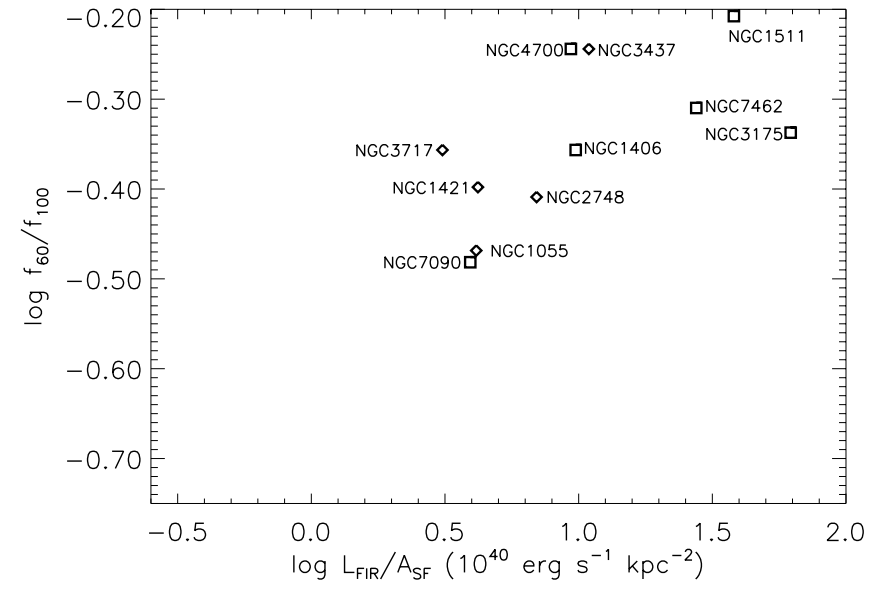

Fig. 38. Plot of the $f_{60} / f_{100}$ FIR flux density ratios vs. the FIR luminosity normalised by the area in which star formation and thus energy input into the ISM takes place, $A_{\mathrm{SF}}$. The open squares represent galaxies where halo emission was clearly detected, diamonds denote systems with marginal or no detections.

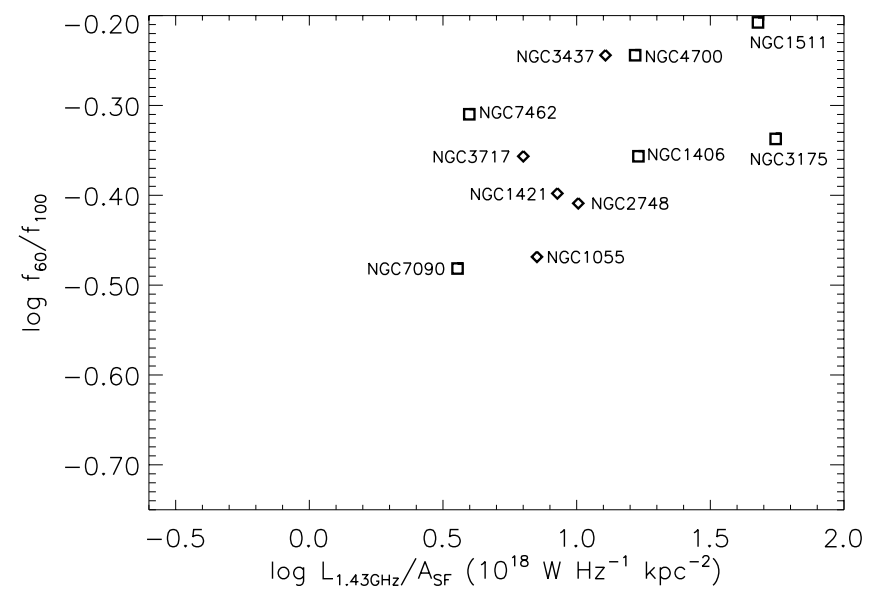

Fig. 39. Plot of the $f_{60} / f_{100}$ FIR flux density ratios vs. the $1.43 \mathrm{GHz}$ radio luminosity normalised by the area in which star formation and thus energy input into the ISM takes place, $A_{\mathrm{SF}}$. The open squares represent galaxies where halo emission was clearly detected, diamonds denote systems with marginal or no detections.

squares). There is a clear trend that halos were detected in those galaxies with the highest $\dot{E} / A_{\mathrm{SF}}$ and $f_{60} / f_{100}$ values, with the notable exception of NGC 3437, which was discussed above. We have selected highly inclined galaxies only for technical reasons. The fact that most, if not all FIR-warm edge-on galaxies have radio halos should apply to galaxies with any inclination angle. We just need to develop tools to find them in more face-on systems.

We have produced the same kind of plot as Fig. 38 based on the total $1.43 \mathrm{GHz}$ radio continuum flux density, as listed in Table 3, which is presented in Fig. 39. The total $1.43 \mathrm{GHz}$ radio luminosity was calculated using the equation $L_{1.43}=4 \pi D^{2} S_{1.43}$, neglecting the term $(1+z)^{1+\alpha}$, because it is not relevant for the low-redshift galaxies studied here (Condon et al. 1990). Because of 
Table 7. Study of halo scale height vs. energy input level.

\begin{tabular}{cccccccc}
\hline Galaxy & $\frac{f_{60}}{f_{100}}$ & $\begin{array}{c}r_{\mathrm{SF}} \\
(" / \mathrm{kpc})\end{array}$ & $\begin{array}{c}A_{\mathrm{SF}} \\
\left(10^{3} \mathrm{kpc}^{2}\right)\end{array}$ & $\begin{array}{c}\mathrm{FIR} \\
\left(10^{-14} \mathrm{~W} \mathrm{~m}^{-2}\right)\end{array}$ & $\begin{array}{c}L_{\mathrm{FIR}} \\
\left(10^{43} \mathrm{erg} \mathrm{s}^{-1}\right)\end{array}$ & $\begin{array}{c}\frac{L_{\mathrm{FIR}}}{A_{\mathrm{SF}}} \\
\left(10^{40} \mathrm{erg} \mathrm{s}^{-1} \mathrm{kpc}^{-2}\right)\end{array}$ & $\begin{array}{c}z_{0, \text { halo }}^{a} \\
\left(\mathrm{kpc}^{2}\right.\end{array}$ \\
\hline NGC 1055 & 0.34 & $236 / 18.3$ & 1.06 & 135.85 & 4.16 & 3.94 & - \\
NGC 1406 & 0.44 & $110 / 7.9$ & 0.20 & 72.74 & 1.93 & 9.75 & 1.66 \\
NGC 1421 & 0.40 & $145 / 21.9$ & 1.50 & 54.43 & 6.30 & 4.20 & - \\
NGC 1511 & 0.62 & $73 / 6.2$ & 0.12 & 124.81 & 4.57 & 38.08 & 1.76 \\
NGC 2748 & 0.39 & $103 / 14.3$ & 0.65 & 45.54 & 4.49 & 6.96 & - \\
NGC 3175 & 0.46 & $45 / 3.5$ & 0.04 & 78.02 & 2.36 & 62.11 & 1.62 \\
NGC 3437 & 0.57 & $96 / 11.9$ & 0.48 & 62.77 & 4.88 & 3.09 & - \\
NGC 3717 & 0.44 & $177 / 23.3$ & 1.71 & 60.24 & 5.29 & 9.35 & 3.06 \\
NGC 4700 & 0.57 & $54 / 6.6$ & 0.14 & 16.67 & 1.30 & 3.93 & 1.77 \\
NGC 7090 & 0.33 & $131 / 7.4$ & 0.17 & 41.76 & 0.68 & 27.54 & 1.82 \\
NGC 7462 & 0.49 & $103 / 7.5$ & 0.18 & 18.07 & 4.93 & 64.50 & - \\
\hline NGC 1569 & 0.96 & $72 / 0.77$ & 0.00186 & 207.20 & 0.12 & & - \\
\hline
\end{tabular}

Note to Table $7:^{a}$ ) 1.425-1.49 GHz measurements only; average values from Table 4.

the strong radio-FIR correlation the two plots look almost identical, although the positions of individual galaxies can shift with respect to their location in Fig. 38. This similarity of the two plots-and thus relations between luminosity and dust temperature-makes our present study and the results by Rossa \& Dettmar (2000) comparable to our earlier investigations (DLG95). As in Fig. 38, the data points in Fig. 39 also indicate a trend of the galaxies with the highest $\dot{E} / A_{\mathrm{SF}}$ values having the highest mean dust temperatures.

A second important result is that all physically small galaxies (with $D_{25} \leq 20 \mathrm{kpc}$; in the present sample NGC 1511, NGC 4700, NGC 7090 and NGC 7462) have prominent radio halos. Their total mass being low, it appears to be easier for CR electrons to escape from the thin disk than in galaxies with higher total masses, $M_{\text {tot }}$. Even NGC 7090, with a moderate mean dust temperature and also a correspondingly moderate energy injection rate $\left(L_{\mathrm{FIR}} / A_{\mathrm{SF}}\right.$; note that NGC 1055 and NGC 7090 have almost equal values, see Table 7 ), can produce a radio halo. Radio halos are visible, because these galaxies are no dwarfs (in which case they might be too light to retain their magnetic fields so that CRs might escape almost lossfree and thereby remain invisible), but intermediate-mass systems.

We note here that the simplifying assumption has been made that all energy contained in CR electrons emitting synchrotron radiation originally arises from the galaxy disks. Lacking images showing details, such an assumption must be made, without any distinction as to where the radio continuum emission comes from. The same assumption is made for the FIR data too (not only by us, but also by Rossa \& Dettmar 2000 and other investigators).

Based on a larger sample, Irwin et al. (1999) did not find a connection between the properties of gaseous halos and the global level of underlying SF activity, using radio continuum images. However, these authors did not distinguish between different object classes contained in their sample due to the small number of galaxies in total. From their sample, NGC 3735 and NGC 4388 should be excluded, because they host Sy-2 nuclei (NED). The existence of a radio halo in NGC 3432, despite its low energy injection rate, can be explained because it is a magellanic irregular with low total mass and thus shallow gravitational potential, similar to the small galaxies in our sample. In addition, NGC 3432 is closely interacting and should therefore be removed from the sample for studies of the dependence of halo properties on the level of underlying SF activity in the galaxy disk. NGC 5433 and IC 562 from the sample by Irwin et al. (1999) should be excluded, as we did above with NGC 5073 and NGC 7541, because their emission distribution is not resolved by the data. Once this target selection is applied, we do see a trend in the data by Irwin et al. (1999) suggesting that the galaxies with the highest $\dot{E} / A_{\mathrm{SF}}$ values have halos, while those with lower energy input levels do not always have halos. Therefore, there is no contradiction in the data by these authors to our hypothesis from the paper by DLG95. Instead, their data support our claim, as do our new results presented here, that late-type spiral galaxies with warm dust do have gaseous halos and that those with the most active SF tend to have the most prominent halos.

\subsubsection{Why is there no direct relationship between $z_{0, \text { halo }}$ and $\dot{E} / A_{\mathrm{SF}}$ ?}

However, trying to go one step further, we find that there is clearly no direct quantitative relationship between the halo scale height, $z_{0 \text {,halo }}$, and the normalised energy input, $\dot{E} / A_{\mathrm{SF}}$, as listed in Table 7 .

In our earlier sample (DLG95) we presented only $L_{*}$ galaxies, i.e. systems near the "knee" of the galaxy luminosity function, with total masses somewhere on the order of $10^{11} M_{\odot}$. There, we found a trend that galaxies with the highest $\mathrm{SF}$ rates in their disks have the most 
prominent halos and that the halos are brightest above the most actively star-forming regions.

The present sample, as indicated above, contains a number of physically small galaxies as well as $L_{*}$ galaxies. This implies that, compared to our earlier study (DLG95), an additional free parameter has entered the equation, namely the different resistence that particles meet when expelled from their birth sites in the disks.

We have therefore tried to normalise the energy input rate not only by the area over which it is distributed, but also by the gravitational potential of the galaxy as measured by the total mass. Also for this quantity we have found no convincing correlation with $z_{0 \text {, halo. This }}$ implies that yet more, or other, factors affect the propagation of CRs out of galaxy disks. In particular, the energy losses that CR electrons undergo on their way out of the disk influence the properties of the observed radio halos: Although predicting different kinds of behaviour, both static and dynamic models of $\mathrm{CR}$ radio halos (Lerche \& Schlickeiser 1981a-c; Werner 1988) predict a dependence of the extent of radio halos on the mean lifetime of the CR electrons. In case of pure diffusive CR propagation the dependence is directly proportional to the diffusion index of the CRs. The influence of convection is expected to increase with increasing energy input. However, at the same time inverse Compton losses become more important with increasing energy input too. This is the reason why no direct dependence is found between the measured scale heights and the level of energy input in the underlying disks.

Significant energy losses of the CR electrons leaving the disk are in agreement with the observed slight steepening of the radio spectral indices (Sect. 3.3).

\subsection{4. rSF vs. radial halo extent}

Based on images with sufficient spatial resolution a direct morphological comparison can be made, as done by us earlier (DLG95), of the radial extent of the radio halo with respect to the radial extent of the actively star-forming part of the disk, $r_{\mathrm{SF}}$. We had found in our previous sample that the radio halo emission breaks off radially near the point where the surface brightness in the disk drops rapidly, i.e. $r_{\mathrm{SF}}$. This implies that halo emission is observed only above the most actively star-forming parts of the galaxy disks. In the present sample, the ATCA data have the highest angular resolution. The effect is visible most clearly in NGC 7090, and also-though to a lesser degree-in NGC 7462 (here especially at $2.45 \mathrm{GHz}$, where the angular resolution is highest). Higher resolution is required to study the same effect in the galaxies observed with the VLA D array, especially in cases like NGC 4700 (cf. Fig. 11). In the case of NGC 3175 the data by Condon et al. (1996) suggest the same behaviour. The extraplanar radio emission is restricted to the radial regime of the central starburst. More details on this will be presented elsewhere.
Despite the relatively good spatial resolution of our data, NGC 1511, with its very smooth emission distribution, shows little of this effect. It comes closest to the ellipsoidal emission distribution that might be expected in case of purely diffusive particle propagation.

\section{Summary and future prospects}

The above results prove that, after exclusion of host galaxies of AGNs, their $f_{60} / f_{100}$ FIR colour (and thus mean dust temperature) is a very successful criterion to select candidate galaxies for searches of radio halos (gaseous halos in general). Sensitive radio continuum imagery is a powerful tool to detect the halo emission.

For the 11 suitable objects which are presented here, we have a detection rate of $55 \%$. Thus, again a substantial fraction of FIR-warm objects, although fainter than those studied by us earlier (DLG95; D98), exhibit halo emission. In some of the remaining cases, especially the galaxies with high $f_{60} / f_{100}$ ratios but no detected radio halos, the angular resolution of our data might not be sufficient to separate thin disk and halo emission. All physically small galaxies in our sample, even some with $f_{60} / f_{100}<0.4$, have radio halos. The measured $z$ scale heights of the radio halos range from about 1.4 to $3.1 \mathrm{kpc}$.

Our results suggest that the galaxies with the highest energy input rates into their disk ISM are the ones that have the most prominent radio halos. However, there is no direct relationship between the halo scale heights and the level of energy input in the underlying disks, because the halo properties far away from the disk planes are dominated by the energy losses of the CR electrons.

To confirm the results obtained here and to improve the angular resolution of the datasets where it limits our investigation, we plan to extend our VLA observations at $1.43 \mathrm{GHz}$ by adding longer baselines from the $\mathrm{C}$ array. This will enable us to study the dependence of the intrinsic radio halo properties on the local level of SF in the underlying disks, as already done for NGC 891 and NGC 4631 (DLG95), in a larger sample.

Acknowledgements. We thank both the ATNF and NRAO for the generous allocation of observing time and the referee, Dr. J. Condon, for fruitful discussions, which helped improve the paper considerably. We thank J. Rossa very much for communicating to us his results of $\mathrm{H} \alpha$ observations prior to publication. M. D. thanks Drs. R. Sault, R. Wark and H. May at the ATNF for their software and computing support and Dr. F. Israel for his kind hospitality at Sterrewacht Leiden, where most of this work was done. Thanks are also due to the VLA analysts for their support during the observations and data reduction. Many thanks to Dr. M. Gotzens-Petr, J. Krist and K. Kraiberg-Knudsen for helping us with the production of the $z$ profiles and to Dr. G. Meurer for the calculation of the distance values. We thank Dr. J. Irwin for making available to us the VLA C array observations of NGC 1421 and Dr. J. Condon for the C array data of NGC 3175. Part of J. L.'s work was performed as part of an ATNF Vacation Scholarship. This research has made use of the NASA Extragalactic Database (NED), whose contributions 
to this paper are gratefully acknowledged. The Digitized Sky Survey was produced at the Space Telescope Science Institute under U.S. Government grant NAG W-2166. The National Geographic Society - Palomar Observatory Sky Atlas (POSSI) was made by the California Institute of Technology with grants from the National Geographic Society.

\section{References}

Baars, J. W. M., Genzel, R., Pauliny-Toth, I. I. K., \& Witzel, A. 1977, A\&A, 61, 99

van den Bergh, S., \& Hazen, M. L. 1988, PASP, 100, 1542

Combes, F. 1987, in Proc. NATO Conf. on Galactic and Extragalactic Star Formation, ed. R. Pudritz, \& M. Fich (Reidel Publ. Co.), 475

Condon, J. J., Yin, Q. F., \& Burstein, D. 1987, ApJS, 65, 543

Condon, J. J., Helou, G., Sanders, D. B., \& Soifer, B. T. 1990, ApJS, 73, 359

Condon, J. J., Helou, G., Sanders, D. B., \& Soifer, B. T. 1996, ApJS, 103, 81

Dahlem, M., Aalto, S., Klein, U., et al. 1990, A\&A, 240, 237

Dahlem, M., Dettmar, R.-J., \& Hummel, E. 1994, A\&A, 290, 384

Dahlem, M., Lisenfeld, U., \& Golla, G. 1995, ApJ, 444, 119 [DLG95]

Dahlem, M. 1997, PASP, 109, 1298

Dahlem, M., Petr, M. G., Lehnert, M. D., Heckman, T. M., \& Ehle, M. 1997, A\&A, 320, 731

Dahlem, M., Weaver, K. A., \& Heckman, T. M. 1998, ApJS, 118, 401 [D98]

Dahlem, M., Ehle, M., \& Ryder, S. D. 2001, A\&A, 371, 45

Dettmar, R.-J. 1992, Fund. Cosm. Phys., 15, 143

Ehle, M., Pietsch, W., Beck, R., \& Klein, U. 1998, A\&A, 329, 39

Fabbiano, G., Kim, D.-W., \& Trinchieri, G. 1992, ApJS, 80, 531
Harnett, J. I., \& Reynolds, J. E. 1985, MNRAS, 215, 247

Heckman, T. M., Armus, L., \& Miley, G. K. 1990, ApJS, 74, 833

Hummel, E., Pedlar, A., van der Hulst, J. M., \& Davies, R. D. 1985, A\&AS, 60, 293

Hummel, E., \& van der Hulst, J. M. 1989, A\&AS, 81, 51

Hummel, E., van der Hulst, J. M., Kennicutt, R. C., \& Keel, W. C. 1990, A\&A, 236, 333

Hummel, E., Beck, R., \& Dettmar, R.-J. 1991a, A\&AS, 87, 309

Hummel, E., Dahlem, M., van der Hulst, J. M., \& Sukumar, S. 1991b, A\&A, 246, 10

Irwin, J. A., English, J., \& Sorathia, B. 1999, AJ, 117, 2102

Lehnert, M. D., \& Heckman, T. M. 1995, ApJS, 97, 89

Leitherer, C., \& Heckman, T. M. 1995, ApJS, 96, 9

Leitherer, C., Schaerer, D., Goldader, J. D., et al. 1999, ApJS, 123,3

Lerche, I., \& Schlickeiser, R. 1981a, ApJS, 47, 33

Lerche, I., \& Schlickeiser, R. 1981b, A\&A, 107, 148

Lerche, I., \& Schlickeiser, R. 1981c, Astroph. Lett., 22, 31

Lesch, H., Biermann, P. L., Crusius, A., et al. 1990, MNRAS, 242,194

Lisenfeld, U., \& Völk, H. J. 2000, A\&A, 354, 423

Lutz, D. 1992, A\&A, 259, 462

Meurer, G. R., Heckman, T. M., Leitherer, C., et al. 1995, AJ, 110,2665

Meurer, G. R., Heckman, T. M., Lehnert, M. D., Leitherer, C., \& Lowenthal, J. 1997, AJ, 114, 54

Rand, R. J. 1996, ApJ, 462, 712

Rossa, J. 2001, Ph.D. Thesis, Rühr-University Bochum, Germany

Rossa, J., \& Dettmar, R.-J. 2000, A\&A, 359, 433

Ryder, S., \& Dopita, M. 1994, ApJ, 430, 142

Tully, R. B. 1988, Nearby Galaxies Catalog (Cambridge University Press)

Werner, W. 1988, A\&A, 201, 1 